\title{
Soft Constrained Model Predictive Control with Robust Stability Guarantees
}

\author{
Melanie N. Zeilinger, Member, IEEE, Manfred Morari, Fellow, IEEE, and Colin N. Jones, Member, IEEE
}

\begin{abstract}
Soft constrained MPC is frequently applied in practice in order to ensure feasibility of the optimization during online operation. Standard techniques offer global feasibility by relaxing state or output constraints, but cannot ensure closedloop stability. This paper presents a new soft constrained MPC approach for tracking that provides stability guarantees even for unstable systems. Two types of soft constraints and slack variables are proposed to enlarge the terminal constraint and relax the state constraints. The approach ensures feasibility of the MPC problem in a large region of the state space, depending on the imposed hard constraints, and stability is guaranteed by design. The optimal performance of the MPC control law is preserved whenever all state constraints can be enforced. Asymptotic stability of all feasible reference steadystates under the proposed control law is shown, as well as inputto-state stability for the system under additive disturbances. The soft constrained method can be combined with a robust MPC approach, in order to exploit the benefits of both techniques. The properties of the proposed methods are illustrated by numerical examples.
\end{abstract}

Index Terms-Soft constraints, Model Predictive Control

\section{INTRODUCTION}

In control systems, there are generally two types of constraints: those originating from physical limitations of the actuators or the system itself, including critical bounds related to, e.g., safe operation of the plant, or constraints derived from desired system specifications. While input constraints can therefore generally not be exceeded, state or output constraints can either be hard if they fall under the first category, or they can be considered soft. Violation may then in practice be tolerated for short time periods, e.g. because of unexpected disturbances. Model predictive control (MPC) is a successful paradigm for the control of constrained systems and offers guaranteed constraint satisfaction as well as stability in closedloop, when all constraints are enforced in the MPC problem [1]. Imposing hard state or output constraints can, however, be overly conservative or render the optimization problem infeasible in closed-loop operation. One possible remedy is to simply remove the constraints for some portion of the prediction horizon until the problem becomes feasible. However, this may lead to large constraint violations in closed-loop, when

M.N. Zeilinger is with the Department of Electrical Engineering and Computer Sciences, UC Berkeley, CA 94720, USA, and the Department of Empirical Inference, Max Planck Institute for Intelligent Systems, 72076 Tübingen, Germany, email: mzeilinger@berkeley.edu.

C.N. Jones is with the Laboratoire d'Automatique, École Polytechnique Fédérale de Lausanne (EPFL), CH-1015 Lausanne, Switzerland, email: colin.jones@epfl.ch.

M. Morari is with the Automatic Control Laboratory, ETH Zurich, $\mathrm{CH}-$ 8092 Zurich, Switzerland, email: morari@ control.ee.ethz.ch.

Manuscript received May 21, 2013; revised December 2, 2013. implementing the first control input of the horizon, without any possibility to tune the amount of violation. A popular approach is a so called soft constrained technique, where state or output constraints are relaxed and the size of the violation is penalized in the cost. While this recovers feasibility of the MPC problem and offers the ability to tune the performance, standard soft constrained MPC schemes generally do not provide stability guarantees.

In this paper we propose a soft constrained linear MPC approach for tracking that guarantees stability even for openloop unstable systems. Although soft constraints are widely used in practical implementations of MPC, this topic has received comparably little attention in the literature. In [2], a condition on the quadratic penalty on the output constraint violation is derived to guarantee stability for single-input single-output systems. In [3], MPC with hard input and soft output constraints is considered and stability is proven for open-loop stable systems by showing that the stability proof in MPC extends to this case. This result also holds for marginally stable systems, if the horizon length is sufficiently long, which is however difficult to choose in practice. The performance of soft constrained MPC for relaxing output constraints was investigated in [4]. The use of exact penalty functions in order to enforce hard constraints when possible is discussed in [5], [6], in which case the stability properties are preserved in the feasible set of the corresponding hard constrained problem. In [7], a Youla parametrization is employed and robust stability of the hard or soft constrained problem is enforced by adding an LMI to the MPC problem. The use of barrier functions to replace constraints presented in [8] is a related idea, however the key difference is that the barrier imposes a penalty inside the constraint set, whereas in soft constrained schemes a penalty is only imposed on the constraint violation. As a result, this approach only provides stability in the feasible set of the corresponding hard constrained problem.

The method proposed in this paper has the advantage that it is conceptually similar to a standard soft constrained technique usually applied in practice, but it also maintains the desirable properties of MPC. Feasibility of the MPC problem is ensured in a large region of the state-space, which depends on the imposed hard constraints. Stability is guaranteed by design, while allowing to tune the system performance and the amount of constraint violation. The method is based on the MPC approach for tracking introduced in [9] and uses a finite horizon with a terminal weight as well as a terminal constraint. All input constraints are hard constraints, while state constraints are softened in two ways. Since a complete relaxation of the terminal constraint leads to a loss of the 
stability properties, we restrict the amount of relaxation by using an enlarged terminal set. All other state constraints are softened by the introduction of two types of slack variables, which is a crucial item for proving stability. The approach allows for any positive definite, convex penalty function on the constraint violation. Here, we include a quadratic and an $l_{1}$ - or $l_{\infty}$-norm penalty in order to allow for better tuning and for exact penalty functions, which preserve the optimal MPC behavior whenever the state constraints can be enforced [4], [5]. The proposed problem setup results in a convex secondorder cone program (SOCP), which can be solved efficiently using, e.g., interior-point methods [10]-[13].

We show that asymptotic stability of all feasible reference steady-states in the absence of disturbances is guaranteed within the feasible set of the soft constrained MPC problem. In addition, input-to-state stability of the proposed scheme under additive disturbances is proven. The robust invariant set, in which input-to-state stability can be guaranteed, depends on the maximum disturbance size. Using the presented soft constrained method, stability can be provided in a potentially much larger set than with a hard constrained method and unexpected disturbances can be tolerated by relaxing state constraints. The soft constrained scheme can also be combined with a robust MPC framework. The advantages of both techniques can be exploited in order to account for a certain disturbance size with a robust design, while dealing with exceeding disturbances by means of soft constraints. We show that the stability results extend to the combined robust and soft constrained approach.

A numerical example demonstrates that the proposed scheme provides feasibility and stability for a large region of the state space and that significant disturbances can be tolerated. Application to a large-scale example shows that the softconstrained MPC problem can be solved with computation times in the millisecond range even for significant problem dimensions.

This paper extends the initial work in [14] to a soft constrained method for tracking and proposes a new combination of the soft constrained scheme with robust MPC, including new theoretical and numerical results. The outline of the paper is as follows: After reviewing some preliminary results in Section II, Section III introduces the problem and the proposed soft constrained MPC formulation for tracking. Asymptotic stability of the nominal system under the resulting control law is proven in Section IV. Section V shows input-to-state stability of the uncertain system under the soft constrained control law as well as the combined robust and soft constrained approach. The properties of the presented approach are illustrated in Section VI by numerical examples.

\section{PReliminaries}

A polyhedron is the intersection of a finite number of halfspaces $P=\{x \mid A x \leq b\}$ and a polytope is a bounded polyhedron. If $A \in \mathbb{R}^{m \times n}$, then $A_{i} \in \mathbb{R}^{n}$ is the vector formed by the $i$ 'th row of $A$. If $b \in \mathbb{R}^{m}$ is a vector, then $b_{i}$ is the $i$ 'th element of $b$. Given a sequence $\mathbf{u} \triangleq\left[u_{0}, \cdots, u_{N-1}\right], u_{j}$ denotes the $j$ 'th element of $\mathbf{u}$. If a sequence depends on a parameter denoted by $\mathbf{u}(x), u_{j}(x)$ denotes its $j$ 'th element. If $x \in \mathbb{R}^{n}$ is a vector and $Q$ is a positive semi-definite matrix, then $\|x\|_{Q}^{2}=x^{T} Q x$ and $[x]_{+}=\max \{0, x\}$ taken elementwise. A function $\gamma: \mathbb{R}_{\geq 0} \rightarrow \mathbb{R}_{\geq 0}$ is of class $\mathcal{K}$ if it is continuous, strictly increasing and $\gamma(0)=0$ [15]. If in addition $\gamma(s) \rightarrow \infty$ as $s \rightarrow \infty$, then it is of class $\mathcal{K}_{\infty}$. A function $\beta: \mathbb{R}_{\geq 0} \times \mathbb{R}_{\geq 0} \rightarrow \mathbb{R}_{\geq 0}$ is of class $\mathcal{K} \mathcal{L}$ if for each fixed $t \geq 0, \beta(\cdot, t)$ is of class $\mathcal{K}$, for each fixed $s \geq 0, \beta(s, \cdot)$ is non-increasing and $\beta(s, t) \rightarrow 0$ as $t \rightarrow \infty$ [15].

Consider the discrete-time uncertain linear system

$$
x(k+1)=A x(k)+B u(k)+w(k), k \in \mathbb{N}
$$

that is subject to the following constraints:

$$
x(k) \in \mathbb{X} \subset \mathbb{R}^{n}, u(k) \in \mathbb{U} \subset \mathbb{R}^{m},
$$

where $x(k)$ is the state, $u(k)$ is the control input and $w(k) \in$ $\mathcal{W} \subset \mathbb{R}^{n}$ is a bounded disturbance at the $k$ 'th sample time. $\mathbb{X} \triangleq\left\{x \mid G_{x} x \leq f_{x}\right\}$ and $\mathbb{U} \triangleq\left\{u \mid G_{u} u \leq f_{u}\right\}$, where $G_{x} \in$ $\mathbb{R}^{p_{x} \times n}, f_{x} \in \mathbb{R}^{p_{x}}$ and $G_{u} \in \mathbb{R}^{p_{u} \times m}, f_{u} \in \mathbb{R}^{p_{u}}$, are polytopic constraints on the states and inputs that each contain the origin in their interior. $\mathcal{W}$ is a convex and compact disturbance set that contains the origin. When it is convenient, we make use of the lighter notation $x^{+}=A x+B u+w$, where $x^{+}$denotes the successor state at the next sampling time. The nominal model of system (1) describes the system considering no disturbance, given by

$$
\bar{x}(k+1)=A \bar{x}(k)+B \bar{u}(k) .
$$

The solution of the uncertain system controlled by the control law $u(k)=\kappa(x(k))$ at sampling time $k$ for the initial state $x(0)$ and for a sequence of disturbances $\mathbf{w}$ is denoted as $\phi_{\kappa}(k, x(0), \mathbf{w})$.

A steady-state and input pair $z_{s} \triangleq\left(x_{s}, u_{s}\right)$ of the nominal system (3) is characterized by the condition $(I-A) x_{s}=B u_{s}$. The constraints limit the set of feasible steady-states to $\mathcal{S} \triangleq$ $\left\{\left(x_{s}, u_{s}\right) \mid\left(x_{s}, u_{s}\right) \in \mathbb{X} \times \mathbb{U},(A-I) x_{s}+B u_{s}=0\right\}$ and in the soft constrained case to $\mathcal{S}^{s} \triangleq\left\{\left(x_{s}, u_{s}\right) \mid u_{s} \in \mathbb{U},(A-I) x_{s}+\right.$ $\left.B u_{s}=0\right\}$. The set of admissible steady-states for tracking is given by $\mathcal{S}^{t r} \triangleq\left\{\left(x_{s}, u_{s}\right) \mid(1+\xi) G_{x} x_{s} \leq f_{x},(1+\xi) G_{u} u_{s} \leq\right.$ $\left.f_{u},(A-I) x_{s}+B u_{s}=0\right\} \subset \mathcal{S}$, where $0<\xi \ll 1$ is a small positive constant, restricting the reference to the interior of the constraints. While the system under consideration may be unstable, it is assumed to satisfy the following standing assumption:

Assumption II.1. The pair (A,B) is stabilizable.

The following standard definitions can be found in [16]:

Definition II.2 ((Robust) positively invariant set). A set $S \subseteq \mathbb{R}^{n}$ is a robust positively invariant (RPI) set of system $x^{+}=f(x)+w$, if $f(x)+w \in S$ for all $x \in S, w \in \mathcal{W}$.

$S$ is called a positively invariant (PI) set of system $x^{+}=f(x)$, if $f(x) \in S$ for all $x \in S$.

Stability of an uncertain system will be analyzed using the framework of input-to-state stability (ISS):

Definition II.3 (Regional ISS [17], [18]). Given an RPI set $\Gamma \subseteq \mathbb{R}^{n}$ containing the origin in its interior, system $x(k+$ $1)=f(x(k))+w(k)$ is Input-to-State Stable (ISS) in $\Gamma$ with 
respect to $w(k) \in \mathcal{W}$, if there exists a $\mathcal{K} \mathcal{L}$-function $\beta$ and a $\mathcal{K}$-function $\gamma$ such that for all initial states $x(0) \in \Gamma$ and for all disturbance sequences $\mathbf{w} \triangleq\left[w_{j}\right]_{j \geq 0}$ with $w_{j} \in \mathcal{W}$ : $\left\|\phi_{\kappa}(k, x(0), \mathbf{w})\right\| \leq \beta(\|x(0)\|, k)+\gamma\left(\left\|\mathbf{w}_{[0, k-1]}\right\|\right) \forall k \geq 0$, where $\left\|\mathbf{w}_{[0, k-1]}\right\| \triangleq \max \left\{\left\|w_{j}\right\|, j \in[0, k-1]\right\}$.

Note that the condition for input-to-state stability reduces to that for asymptotic stability, if $w_{j}=0$ for all $j \geq 0$.

Theorem II.4 (Regional ISS [19], [20]) Let $\Gamma$ be an RPI set for system $x(k+1)=f(x(k))+w(k)$ and $S \subseteq \Gamma$ be a compact set, both including the origin as an interior point. If there exists a function $V: \mathbb{R}^{n} \rightarrow \mathbb{R}_{+}$, suitable $\mathcal{K}_{\infty}$-class functions $\alpha_{1}, \alpha_{2}, \alpha_{3}$ and a $\mathcal{K}$-class function $\gamma$ such that:

$$
\begin{aligned}
& V(x) \geq \alpha_{1}(\|x\|) \forall x \in \Gamma, \\
& V(x) \leq \alpha_{2}(\|x\|) \forall x \in S, \\
& V(f(x)+w)-V(x) \leq-\alpha_{3}(\|x\|)+\gamma(\|w\|) \\
& \quad \forall x \in \Gamma, w \in \mathcal{W},
\end{aligned}
$$

$V(\cdot)$ is called an ISS Lyapunov function in $\Gamma$ and the system $x(k+1)=f(x(k))+w(k)$ is ISS in $\Gamma$ with respect to $w \in \mathcal{W}$.

\section{A. MPC for Tracking Piecewise Constant References}

We consider the problem of tracking a given reference steady-state $z_{r} \triangleq\left(x_{r}, u_{r}\right) \in \mathcal{S}^{t r}$ starting from a given initial state $x$. This work employs the tracking formulation introduced in [9] as the basis for the proposed soft constrained scheme, offering recursive feasibility and an enlarged region of attraction compared to the standard approach of applying a change of variables [20], [21]. An artificial steady-state is introduced, which may deviate from the desired reference if the latter is not a feasible target from the current state, and can be seen as an artificial set point that is simultaneously steered to the reference. The cost is then designed for tracking the artificial steady-state, where a penalty term on the deviation between the artificial and the real steady-state ensures convergence to the desired reference. The resulting nominal MPC problem for tracking $\mathbb{P}_{N}\left(x, z_{r}\right)$ is given by:

$$
\begin{aligned}
V_{N}\left(\mathbf{x}, \mathbf{u}, z_{s}, z_{r}\right) \triangleq & \sum_{i=0}^{N-1} l\left(x_{i}-x_{s}, u_{i}-u_{s}\right)+V_{f}\left(x_{N}-x_{s}\right) \\
& +V_{o}\left(x_{s}-x_{r}, u_{s}-u_{r}\right) \\
V_{N}^{*}\left(x, z_{r}\right) \triangleq \min _{\mathbf{x}, \mathbf{u}, z_{s}} & V_{N}\left(\mathbf{x}, \mathbf{u}, z_{s}, z_{r}\right) \\
\text { s.t. } \quad & x_{0}=x \\
& x_{i+1}=A x_{i}+B u_{i}, \\
& \left(x_{i}, u_{i}\right) \in \mathbb{X} \times \mathbb{U} \\
& x_{N} \in \mathcal{X}_{f}\left(x_{s}, u_{s}\right) \\
& \left(x_{s}, u_{s}\right) \in \mathcal{S}
\end{aligned}
$$

for $i=0, \ldots, N-1$, where $\mathbf{x}=\left[x_{0}, x_{1}, \cdots, x_{N}\right]$ and $\mathbf{u}=\left[u_{0}, \cdots, u_{N-1}\right]$ denote the state and input sequences, the stage cost is defined as $l(x, u) \triangleq\|x\|_{Q}^{2}+\|u\|_{R}^{2}, V_{f}(x) \triangleq\|x\|_{P}^{2}$ is a terminal penalty function and $Q, R$ and $P$ are symmetric positive definite matrices. In this tracking formulation $z_{s}=\left(x_{s}, u_{s}\right)$ denotes the artificial steady-state and input pair,
$X_{f}\left(x_{s}, u_{s}\right)$ is a compact terminal set for tracking that is parameterized by the steady-state, and $V_{o}(\cdot, \cdot): \mathbb{R}^{n} \times \mathbb{R}^{m} \rightarrow \mathbb{R}_{+}$ is a positive definite cost on the tracking offset. We refer to [9], [22] for more details on this approach.

Problem $\mathbb{P}_{N}\left(x, z_{r}\right)$ implicitly defines the set of feasible control sequences $\mathcal{U}_{N}\left(x, z_{s}\right) \triangleq\{\mathbf{u} \mid \exists \mathbf{x}$ s.t. $(5 \mathrm{c})-(5 \mathrm{f})$ hold $\}$ and feasible initial states $\mathcal{X}_{N} \triangleq\left\{x \mid \exists z_{s} \in \mathcal{S}\right.$ s.t. $\left.\mathcal{U}_{N}\left(x, z_{s}\right) \neq \emptyset\right\}$. Note that the feasible set is independent of the given reference. The resulting MPC control law for tracking is given in a receding horizon fashion by

$$
\kappa\left(x, z_{r}\right)=u_{0}^{*}\left(x, z_{r}\right),
$$

where $\mathbf{u}^{*}\left(x, z_{r}\right)$ is the optimal solution to Problem $\mathbb{P}_{N}\left(x, z_{r}\right)$.

Assumption II.5. It is assumed that for any given $\left(x_{s}, u_{s}\right) \in$ $\mathcal{S}, V_{f}\left(x-x_{s}\right)$ is a Lyapunov function in $\mathcal{X}_{f}\left(x_{s}, u_{s}\right)$ and that $\mathcal{X}_{f}\left(x_{s}, u_{s}\right)$ is a PI set for the nominal system (3) under the local control law for tracking $\kappa_{f}(x)=K\left(x-x_{s}\right)+u_{s}$, which can be stated as the following conditions:

$$
\begin{aligned}
\mathrm{A} 1: & V_{f}\left((A+B K)\left(x-x_{s}\right)\right)-V_{f}\left(x-x_{s}\right) \\
& \leq-l\left(x-x_{s}, K\left(x-x_{s}\right)\right) \forall x \in \mathcal{X}_{f}\left(x_{s}, u_{s}\right) \\
\mathrm{A} 2: & \mathcal{X}_{f}\left(x_{s}, u_{s}\right) \subseteq \mathbb{X}, A x+B \kappa_{f}(x) \in \mathcal{X}_{f}\left(x_{s}, u_{s}\right), \\
& \kappa_{f}(x) \in \mathbb{U} \forall x \in \mathcal{X}_{f}\left(x_{s}, u_{s}\right) .
\end{aligned}
$$

Theorem II.6 (Nominal stability under $\kappa\left(x, z_{r}\right)$ [9])

Let $\left(x_{r}, u_{r}\right) \in \mathcal{S}^{t r}$ be a given reference steady-state. $x_{r}$ is asymptotically stable for the closed-loop system $x^{+}=A x+B \kappa\left(x, z_{r}\right)$ with region of attraction $\mathcal{X}_{N}$.

The extension of this tracking scheme to a robust tubebased MPC framework for systems with bounded additive disturbances of the form (1) was considered in [23].

\section{Soft Constrained MPC - Problem Setup}

This paper develops a soft constrained MPC method based on the tracking formulation $\mathbb{P}_{N}\left(x, z_{r}\right)$, which provides stability guarantees in the presence of soft constraints. We first briefly discuss in the following why the stability properties are lost using a standard soft constrained technique, and then present the new formulation.

\section{A. Problem Statement}

A commonly applied approach is to relax all state constraints by the introduction of slack variables $\epsilon_{i}$ and to minimize the amount of constraint violation by including penalty functions on the slack variables in the MPC cost, i.e. to replace $x_{i} \in \mathbb{X}$ in (5e) with $G_{x} x_{i} \leq f_{x}+\epsilon_{i}$ and to add $\sum_{i=0}^{N-1} l_{\epsilon}\left(\epsilon_{i}\right)$ to the cost function in (5b), where $\epsilon_{i} \geq 0$ and $l_{\epsilon}$ is a positive definite function. Stability would be preserved in this case when imposing a terminal set, in which all state and input constraints are satisfied. Note that the method in [33] can be considered as an approach with a hard terminal constraint, but since the terminal set is $\mathbb{R}^{n}$ for stable systems, global stability can be shown. For marginally stable or unstable systems as considered in this paper, a hard terminal constraint represents a significant limitation. In order to ensure feasibility of the MPC problem in a large region of the state space, an extremely long prediction horizon would have to be chosen or it would 
have to be adapted online; both approaches are undesirable for implementation. Similarly, stability would be guaranteed by using an infinite horizon, which is however intractable in the presence of additional hard constraints.

If the terminal constraint is relaxed by a slack variable that is minimized in the cost, the stability guarantee is lost even in the nominal case. The stability proof employing the optimal MPC cost as a Lyapunov function fails for two possible reasons. If the terminal state is outside the region where a control law stabilizing the unconstrained system is feasible, no input sequence is available for proving a decrease in the cost. If the local control law satisfies the input constraints, but the state constraints are violated, a decrease in the cost can no longer be guaranteed due to the addition of the slack penalties to the cost function.

\section{B. Soft Constrained MPC Problem Formulation}

A stability guarantee by means of the standard stability proof in MPC has to be sacrificed in exchange for a complete relaxation of the terminal constraint. In this work, we therefore propose to use a restricted relaxation by means of an enlarged terminal set that enforces only the input constraints. In addition, two different types of slack variables are employed, which will be key in proving (input-to-state) stability in a large feasible set. The proposed soft constrained MPC problem $\mathbb{P}_{N}^{s}\left(x, z_{r}\right)$ is given by:

Problem $\mathbb{P}_{N}^{s}\left(x, z_{r}\right)$ (Soft constrained MPC problem)

$$
\begin{aligned}
V_{N}^{s}\left(\mathbf{x}, \mathbf{u}, z_{s}, \boldsymbol{\epsilon}, z_{r}\right) \triangleq & V_{N}\left(\mathbf{x}, \mathbf{u}, z_{s}, z_{r}\right)+l_{\epsilon}\left(\epsilon_{s}\right)+\sum_{i=0}^{N-1} l_{\epsilon}\left(\epsilon_{i}+\epsilon_{s}\right) \\
V_{N}^{s *}\left(x, z_{r}\right)=\min _{\mathbf{x}, \mathbf{u}, z_{s}, \boldsymbol{\epsilon}} & V_{N}^{s}\left(\mathbf{x}, \mathbf{u}, z_{s}, \boldsymbol{\epsilon}, z_{r}\right) \\
\text { s.t. } \quad & x_{0}=x \\
& x_{i+1}=A x_{i}+B u_{i}, \\
& G_{u} u_{i} \leq f_{u} \\
& G_{x} x_{i} \leq f_{x}+\epsilon_{s}+\epsilon_{i}, \\
& x_{N} \in \mathcal{E}_{f}^{s}\left(x_{s}, u_{s}\right), \\
& \left(x_{s}, u_{s}\right) \in \mathcal{S}^{s}, \\
& (1+\xi) G_{x} x_{s} \leq f_{x}+\epsilon_{s}, \\
& c\left\|x_{N}-x_{s}\right\|_{T} \leq f_{x}+\epsilon_{s}-G_{x} x_{s} \\
& \epsilon_{i} \geq 0, \epsilon_{s} \geq 0
\end{aligned}
$$

for $i=[0, \ldots, N-1]$, where $\epsilon=\left[\epsilon_{0}, \ldots, \epsilon_{N-1}, \epsilon_{s}\right]$ are the slack variables corresponding to the state sequence $\mathbf{x}$. The offset cost is defined as $V_{o}\left(x_{s}-x_{r}, u_{s}-u_{r}\right)=\rho_{x} \| x_{s}-$ $x_{r}\left\|_{p}+\rho_{u}\right\| u_{s}-x_{r} \|_{p}$ and the penalty function on the slack variables is taken as $l_{\epsilon}(\epsilon)=\|\epsilon\|_{S}^{2}+\rho_{\epsilon}\|\epsilon\|_{p}$, where $S$ is a symmetric positive semi-definite matrix, $p \in\{1, \infty\}$, and $\rho_{x}, \rho_{u}, \rho_{\epsilon} \in \mathbb{R}_{+}$are positive constant weights. We use an invariant ellipsoidal terminal set, given by

$$
\mathcal{E}_{f}^{s}\left(x_{s}, u_{s}\right) \triangleq\left\{x \mid\left\|x-x_{s}\right\|_{T}^{2} \leq 1-r\left(x_{s}, u_{s}\right)\right\},
$$

where $T \in \mathbb{R}^{n \times n}$ is a symmetric positive definite matrix and $r: \mathcal{S}^{s} \rightarrow[0,1)$ is a quadratic positive definite function. $\xi \in$
$\mathbb{R}_{+}$is a small positive constant and $c \in \mathbb{R}^{p_{x}}$ is a constant vector with $c_{i} \triangleq\left\|T^{-\frac{1}{2}} G_{x, i}^{T}\right\|_{2} \forall i=1, \ldots, p_{x}$.

The modifications introduced in Problem $\mathbb{P}_{N}^{s}\left(x, z_{r}\right)$ are explained in the following sections and are illustrated in Figure 1.

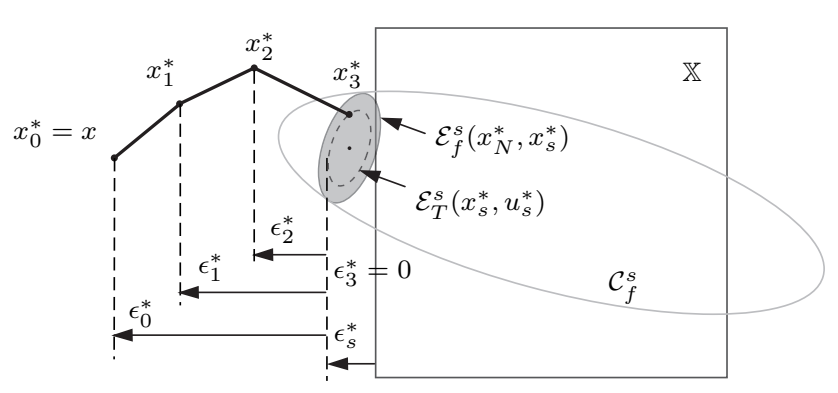

Fig. 1. Illustration of the optimal slack variables $\epsilon_{s}^{*}, \epsilon_{i}^{*}, i=0,1,2,3$, the terminal set $\mathcal{E}_{f}^{s}\left(x_{s}^{*}, u_{s}^{*}\right)$, the scaled terminal set $\mathcal{E}_{T}^{s}\left(x_{N}^{*}, x_{s}^{*}\right)$ and enlarged terminal set $\mathcal{C}_{f}^{s}$ for an initial state $x$ outside $\mathbb{X}$.

Problem $\mathbb{P}_{N}^{s}\left(x, z_{r}\right)$ implicitly defines the set of feasible control sequences $\mathcal{U}_{N}^{s}\left(x, z_{s}\right)=\{\mathbf{u} \mid \exists$ x s.t. $(7 \mathrm{~b})-(7 \mathrm{~d}),(7 \mathrm{f})$ hold $\}$ and feasible initial states $\mathcal{X}_{N}^{s} \triangleq\left\{x \mid \exists z_{s} \in \mathcal{S}^{s}\right.$ s.t. $\mathcal{U}_{N}^{s}\left(x, z_{s}\right) \neq$ $\emptyset\}$. For a given state $x \in \mathcal{X}_{N}^{s}$ and reference $z_{r} \in \mathcal{S}^{t r}$, Problem $\mathbb{P}_{N}^{s}\left(x, z_{r}\right)$ results in a convex second order cone program (SOCP) and its solution yields the optimal control sequence $\mathbf{u}^{s *}\left(x, z_{r}\right)$. Note that SOCPs can be efficiently solved using, e.g., interior-point methods [10]-[13]. The implicit optimal soft constrained MPC control law is then given in a receding horizon fashion by

$$
\kappa^{s}\left(x, z_{r}\right) \triangleq u_{0}^{s *}\left(x, z_{r}\right) .
$$

1) Relaxation of the Terminal Constraint: The terminal set for tracking in (7f) is relaxed by two effects: i) by allowing the artificial set point to move to any steady-state $\left(x_{s}, u_{s}\right) \in \mathcal{S}^{s}$ satisfying only the input constraints, ii) by allowing the state constraints to be violated in the terminal set $\mathcal{E}_{f}^{s}\left(x_{s}, u_{s}\right)$. This results in an enlarged terminal set $\mathcal{C}_{f}^{s} \triangleq\left\{x \mid \exists\left(x_{s}, u_{s}\right) \in\right.$ $\mathcal{S}^{s}$ s.t. $\left.x \in \mathcal{E}_{f}^{s}\left(x_{s}, u_{s}\right)\right\}$ for the MPC problem, given by the set of all states $x$, for which there exists a steady-state such that the terminal constraint is satisfied.

Assumption III.1. For any given $\left(x_{s}, u_{s}\right) \in \mathcal{S}^{s}, \mathcal{E}_{f}^{s}\left(x_{s}, u_{s}\right)$ is a PI set under the local control law $\kappa_{f}^{s}(x)=K\left(x-x_{s}\right)+u_{s}$ satisfying the following conditions:

$$
\begin{aligned}
\text { A3: } & \kappa_{f}^{s}(x) \in \mathbb{U} \forall x \in \mathcal{E}_{f}^{s}\left(x_{s}, u_{s}\right) \\
\mathrm{A} 4: & \left\|A x+B \kappa_{f}^{s}(x)-x_{s}\right\|_{T}^{2}=\left\|(A+B K)\left(x-x_{s}\right)\right\|_{T}^{2} \\
& \leq\left\|x-x_{s}\right\|_{T}^{2} .
\end{aligned}
$$

It is further assumed that the set $\mathcal{C}_{f}^{s}$ is compact.

Note that compared to condition A2 in Assumption II.5, condition A3 only enforces the input constraints. Condition A4 is slightly stronger than set invariance and is required for proving stability in Section IV. If $K$ is taken as the infinite horizon LQR control law, which is a common choice in MPC, a matrix $T$ satisfying condition A4 is, e.g., given by the solution to the discrete-time algebraic Riccati equation. Compactness of $\mathcal{C}_{f}^{s}$ is required to ensure boundedness of the 
feasible set and in turn uniform continuity of the optimal value function $V_{N}^{s *}\left(x, z_{r}\right)$ (Lemma V.1). It can be easily satisfied by imposing a large upper bound on the steady-state $x_{s}$.

Lemma III.2 A matrix $T$ and function $r$ defining the invariant ellipsoidal target set $\mathcal{E}_{f}^{s}\left(x_{s}, u_{s}\right)$ in (8) such that Assumption III.1 is satisfied can be computed by solving a convex linear matrix inequality (LMI).

Proof: See appendix.

Remark III.3. The amount, by which the terminal set can be enlarged depends on $\mathcal{S}^{s}$. An increase can only be achieved if the reference steady-state is not the only steady-state, i.e $x_{s}$ can differ from $x_{r}$, and the imposed hard constraints are not limiting the size of the terminal set to be always contained in the state constraints.

Remark III.4. The combination of moving the artificial steady-state and neglecting state constraints offers a significant increase of the terminal set, which is the reason for choosing the tracking formulation [9]. The proposed scheme could also be applied to a standard MPC formulation by allowing state constraints to be violated in the terminal set, which would, however, result in a smaller terminal and hence feasible set.

2) Slack Variables: We now explain the crucial item in the proposed soft constrained scheme, the slack variables $\epsilon_{s}$ and $\epsilon_{i}$ that are used to soften all state constraints:

- $\epsilon_{s}$ represents the amount of constraint relaxation that is necessary in order to include the ellipsoid $\mathcal{E}_{T}^{s}\left(x_{N}, x_{s}\right)$ for a particular value of $\left(x_{s}, u_{s}\right)$ into the relaxed state constraints, where

$$
\mathcal{E}_{T}^{s}\left(x_{N}, x_{s}\right) \triangleq\left\{x \mid\left\|x-x_{s}\right\|_{T}^{2} \leq\left\|x_{N}-x_{s}\right\|_{T}^{2}\right\}
$$

is a scaling of the ellipsoidal terminal set $\mathcal{E}_{f}^{s}\left(x_{s}, u_{s}\right)$ containing $x_{N}$ on its boundary. This can be expressed as

$\max _{x}\left\{G_{x, i} x \mid\left\|x-x_{s}\right\|_{T}^{2} \leq\left\|x_{N}-x_{s}\right\|_{T}^{2}\right\} \leq f_{x, i}+\epsilon_{s, i}$

$\forall i=1, \ldots, p_{x}$, resulting in the following condition [24]:

$$
\left\|T^{-\frac{1}{2}} G_{x, i}^{T}\right\|_{2}\left\|T^{\frac{1}{2}}\left(x_{N}-x_{s}\right)\right\|_{2} \leq f_{x, i}+\epsilon_{s, i}-G_{x, i} x_{s}
$$

$\forall i=1, \ldots, p_{x}$, which corresponds to (7i) with $c_{i}=$ $\left\|T^{-\frac{1}{2}} G_{x, i}^{T}\right\|_{2} \forall i=1, \ldots, p_{x}$ and is a collection of $p_{x}$ convex second order cone constraints.

- $\epsilon_{i}$ in (7e) represents the additional constraint violation of each state $x_{i}$ for $i=0, \ldots, N-1$ with respect to the state constraints relaxed by $\epsilon_{s}$.

The use of the slack variable $\epsilon_{s}$ defined by (7i) ensures that the terminal state, which is contained in $\mathcal{E}_{T}^{s}\left(x_{N}, x_{s}\right)$, will lie inside the state constraints relaxed by the amount $\epsilon_{s}$ and will not require a further relaxation of the state constraints, i.e. $\epsilon_{N}=0$, where $\epsilon_{N}$ is the slack variable of the terminal state defined by $G_{x} x_{N} \leq f_{x}+\epsilon_{s}+\epsilon_{N}$. This provides feasibility of the shifted sequence using the shifted slack variables with the last slack variable being zero. Constraint (7h) additionally enforces that the steady-state $x_{s}$ always has to lie in the interior of the constraints relaxed by $\epsilon_{s}$ by an amount $\xi$, which is a userspecified small, positive parameter. For a state that is close to the artificial steady-state, this ensures that the steady-state can always be shifted towards the reference without increasing the slack variables.

As will be shown in Section IV, these items provide that the optimal cost function is still a Lyapunov function and are hence crucial for proving stability of the proposed soft constrained MPC scheme.

Remark III.5. By Assumption III.1, the set $\mathcal{E}_{T}^{s}\left(x_{N}, x_{s}\right)$ is a positively invariant set under the local control law $\kappa_{f}^{s}(x)$.

Remark III.6. Defining $\epsilon_{s}$ by the inclusion of $\mathcal{E}_{f}^{s}\left(x_{s}, u_{s}\right)$ into the relaxed state constraints would also provide $\epsilon_{N}=0$ and would allow for proving asymptotic stability. We have chosen $\mathcal{E}_{T}^{s}\left(x_{N}, x_{s}\right)$ here, since it results in a slack closer to the actual constraint violation of the terminal state.

Remark III.7. While a strictly positive value of $\xi$ in constraint (7h) is required to prove stability of the closed-loop system (Lemma IV.2), the particular choice is not crucial and for $\xi \ll 1$, it will have a negligible or no effect on the system behavior.

Remark III.8 (Hard state constraints). For ease of notation, we assume the relaxation of all state constraints except the terminal constraint in $\mathbb{P}_{N}^{s}\left(x, z_{r}\right)$. However, the results directly extend to the case where some of the state constraints are considered as hard constraints with only minor notational changes.

3) Penalty Functions: A penalty function on the slack variables is included in the cost in (7a), in order to minimize the constraint violation and to ensure satisfaction of the state constraints whenever possible. The penalty can be chosen as in standard soft constrained schemes. In the proposed formulation, we include a quadratic penalty, which is often preferable for tuning the constraint violation [4], and an $l_{1}$ or $l_{\infty}$-norm penalty, in order to allow for exact penalty functions. It is well-known that, when the weights on the $l_{1}$ or $l_{\infty}$-norms are sufficiently large and there exists a feasible solution to the hard constrained problem $\mathbb{P}_{N}\left(x, z_{r}\right)$, then the optimal solution to the soft constrained problem $\mathbb{P}_{N}^{s}\left(x, z_{r}\right)$ corresponds to that of the hard constrained problem [5], [6], [25]. Note that an $l_{1}$ or $l_{\infty}$-norm is also used in the offset cost for penalizing the deviation of the artificial from the reference steady-state, in order to enforce the reference as the target point if it is feasible [22].

Remark III.9. The results presented in this paper hold for any positive definite, convex penalty function on the slack variables, i.e. the quadratic penalty could also be omitted. Note that the linear penalties can be simplified for implementation. The $l_{1}$-norm can directly be replaced with the sum of the slack variables, and the $l_{\infty}$-norm can be formulated by using a single slack variable for the constraint relaxation at each stage that is then penalized in the cost.

\section{Soft Constrained MPC Properties}

The soft constrained formulation $\mathbb{P}_{N}^{s}\left(x, z_{r}\right)$ enlarges the feasible set compared to the hard constrained problem since 
$\mathcal{X}_{N} \subseteq \mathcal{X}_{N}^{s}$. By selecting the prediction horizon accordingly, it can be chosen to cover any polytopic region of interest up to the maximum stabilizable set for the input-constrained system, i.e. all initial states for which there exists a feasible input at all times such that the state converges to the origin without considering the state constraints.

In the following sections, we demonstrate how the introduction of the previously described components allows us to show that:

1) The optimal cost function $V_{N}^{s *}\left(x, z_{r}\right)$ is a Lyapunov function and all reference steady-states $z_{r} \in \mathcal{S}^{t r}$ are asymptotically stable for the controlled nominal system with an enlarged region of attraction compared to a standard nominal MPC method (Section IV).

2) The reference steady-state is ISS for the controlled system under additive disturbances using the proposed soft constrained method as well as a combined robust and soft constrained approach (Section V). The region of attraction is enlarged compared to a pure robust MPC approach considering the same disturbance size, which is demonstrated by numerical examples (Section VI).

\section{NOMINAL StabiLity}

In the following, we prove that the resulting optimal soft constrained control law $\kappa^{s}\left(x, z_{r}\right)$ in (9) asymptotically stabilizes the nominal system in (3) in the enlarged PI set $\mathcal{X}_{N}^{s}$. For this, we show in three steps that the optimal cost function of the soft-constrained MPC problem $V_{N}^{s *}\left(x, z_{r}\right)$ is a Lyapunov function.

Lemma IV.1 Let $\quad \mathbf{u}^{s *}\left(x, z_{r}\right), \quad \mathbf{x}^{s *}\left(x, z_{r}\right), \quad x_{s}^{s *}\left(x, z_{r}\right)$, $u_{s}^{s *}\left(x, z_{r}\right), \epsilon^{s *}\left(x, z_{r}\right)$ be the optimizer of $\mathbb{P}_{N}^{s}\left(x, z_{r}\right)$ for some $x \in \mathcal{X}_{N}^{s}$ and reference steady-state $z_{r} \in \mathcal{S}^{\text {tr }}$ and let $x^{+}=A x+B \kappa^{s}\left(x, z_{r}\right)$. The shifted control sequence

$$
\mathbf{u}^{\text {shift }}=\left[u_{1}^{s *}\left(x, z_{r}\right), \ldots, u_{N-1}^{s *}\left(x, z_{r}\right), \tilde{u}\left(x, z_{r}\right)\right],
$$

with $\tilde{u}\left(x, z_{r}\right)=K\left(x_{N}^{s *}(x)-x_{s}^{s *}\left(x, z_{r}\right)\right)+u_{s}^{s *}\left(x, z_{r}\right)$ is feasible for $\mathbb{P}_{N}^{s}\left(x^{+}, z_{r}\right)$ with steady-state $z_{s}^{\text {shift }}=z_{s}^{s *}\left(x, z_{r}\right)$ and slack variables $\boldsymbol{\epsilon}^{\text {shift }}=\left[\epsilon_{1}^{s *}\left(x, z_{r}\right), \ldots, \epsilon_{N-1}^{s *}\left(x, z_{r}\right), 0, \epsilon_{s}^{s *}\left(x, z_{r}\right)\right]$ and

$$
\begin{aligned}
& V_{N}^{s *}\left(x^{+}, z_{r}\right)-V_{N}^{s *}\left(x, z_{r}\right) \\
& \leq-l\left(x-x_{s}^{s *}\left(x, z_{r}\right), u_{0}^{s *}\left(x, z_{r}\right)-u_{s}^{s *}\left(x, z_{r}\right)\right) .
\end{aligned}
$$

Proof: For brevity, we drop the dependence on $\left(x, z_{r}\right)$. Feasibility of $\mathbf{u}^{\text {shift }}$ for $\mathbb{P}_{N}^{s}\left(x^{+}, z_{r}\right)$ with $z_{s}^{\text {shift }}$ and $\boldsymbol{\epsilon}^{\text {shift }}$ follows from feasibility of $\mathbf{u}^{s *}, x_{s}^{s *}, u_{s}^{s *}, \boldsymbol{\epsilon}^{s *}$ at $x$ and positive invariance of $\mathcal{E}_{T}^{s}\left(x_{N}^{s *}, x_{s}^{s *}\right) . \epsilon_{N}^{s *}=0$ results from the fact that $x_{N}^{s *} \in \mathcal{E}_{T}^{s}\left(x_{N}^{s *}, x_{s}^{s *}\right)$ and the definition of the slack variables in (7). (12) then follows from A1 in Assumption II.5 and standard arguments in MPC.

Lemma IV.1 implies that the closed-loop system converges to $x_{s}^{s *}$. In order to achieve asymptotic convergence to the reference $x_{r}$, we have to show that $x_{s}^{s *}$ simultaneously converges to $x_{r}$. We first state a lemma showing that if the state is closer to the artificial steady-state $x_{s}^{s *}$ than some fraction of the distance between the artificial and the target steady-state $x_{r}$, then we can move the artificial steady-state towards $x_{r}$, while providing a decrease in the cost using the auxiliary control law. This result will then allow us to prove one of the main results of this paper in Theorem IV.3 and show asymptotic stability of $x_{r}$ for the closed-loop system under the proposed soft constrained MPC control law.

Lemma IV.2 Let $\left(x_{s}^{a}, u_{s}^{a}\right)$ be a steady-state, $\mathbf{u}^{a}, \mathbf{x}^{a}$ the input and state sequence generated by applying the auxiliary control law $\kappa_{f}^{s}(x)=u_{s}^{a}+K\left(x-x_{s}^{a}\right)$ starting from $x_{0}^{a}$ and let $\boldsymbol{\epsilon}^{a}$ be the associated minimal slacks. Denote $x_{s, \alpha}^{a}=\alpha x_{s}^{a}+(1-$ $\alpha) x_{r}, u_{s, \alpha}^{a}=\alpha u_{s}^{a}+(1-\alpha) u_{r}$. There exist constants $\delta>0$ and $\alpha \in(0,1)$ such that $\left\|x_{0}^{a}-x_{s}^{a}\right\|_{P} \leq(1-\alpha)\left\|x_{s}^{a}-x_{r}\right\|_{P} \leq \delta$ implies that

1) the slacks $\boldsymbol{\epsilon}_{\alpha}^{a}=\boldsymbol{\epsilon}^{a}$ are feasible for $\mathbf{x}^{a}$ and $x_{s, \alpha}^{a}$,

2) $V_{N}\left(\mathbf{x}^{a}, \mathbf{u}^{a}, z_{s, \alpha}^{a}, z_{r}\right)-V_{N}\left(\mathbf{x}^{a}, \mathbf{u}^{a}, z_{s}^{a}, z_{r}\right)$ $\leq-(1-\alpha)^{2}\left\|x_{s}^{a}-x_{r}\right\|_{P}^{2}$,

and therefore

$$
\begin{aligned}
& V_{N}^{s}\left(\mathbf{x}^{a}, \mathbf{u}^{a}, z_{s, \alpha}^{a}, \boldsymbol{\epsilon}_{\alpha}^{a}, z_{r}\right)-V_{N}^{s}\left(\mathbf{x}^{a}, \mathbf{u}^{a}, z_{s}^{a}, \boldsymbol{\epsilon}^{a}, z_{r}\right) \\
& \leq-(1-\alpha)^{2}\left\|x_{s}^{a}-x_{r}\right\|_{P}^{2} .
\end{aligned}
$$

Proof: The result is shown by proving 1) and 2) separately. For 1), we will prove that there exists a $\delta>0$ such that $\epsilon_{s, \alpha}^{a}=\epsilon_{s}^{a}$ is a feasible choice for any $\underline{\alpha}_{1} \triangleq$ $\left(\left\|x_{s}^{a}-x_{r}\right\|_{P}-\delta\right) /\left\|x_{s}^{a}-x_{r}\right\|_{P} \leq \alpha<1$. Feasibility of $\epsilon_{i, \alpha}^{a}=\epsilon_{i}^{a}$ then follows from the use of the same state sequence. For 2), it is shown that the condition holds for $\underline{\alpha}_{2} \leq \alpha<1$, i.e. both 1) and 2) hold for $\max \left\{\underline{\alpha}_{1}, \underline{\alpha}_{2}\right\} \leq \alpha<1$. Note that 2) is independent of the soft constrained formulation and can be shown as in the hard constrained case in [26]. The proof is included in the appendix for completeness.

Proof of 1): Let $A_{K} \triangleq A+B K$. Using the constraints on $\epsilon_{s}^{a}$, we can derive the following two conditions on $\epsilon_{s, \alpha}^{a}$. From (7h), $(1+\xi) G_{x} x_{s}^{a} \leq f_{x}+\epsilon_{s}^{a}$ and recalling that $(1+\xi) G_{x} x_{r} \leq f_{x}$ :

$$
\begin{aligned}
& (1+\xi) G_{x} x_{s, \alpha}^{a} \\
& \leq \alpha\left(f_{x}+\epsilon_{s}^{a}\right)+(1-\alpha) f_{x} \leq f_{x}+\alpha \epsilon_{s}^{a} \leq f_{x}+\epsilon_{s, \alpha}^{a} .
\end{aligned}
$$

By Assumption III.1 and using $T \preceq \gamma^{2} P$, for some $\gamma \geq 1$, we have that $\left\|x_{N}^{a}-x_{s}^{a}\right\|_{T}^{2} \leq\left\|x_{0}^{a}-x_{s}^{a}\right\|_{T}^{2} \leq \gamma^{2}\left\|x_{0}^{a}-x_{s}^{a}\right\|_{P}^{2} \leq \gamma^{2} \delta^{2}$. From (7i) we then obtain:

$$
\begin{aligned}
& c\left\|x_{N}^{a}-x_{s, \alpha}^{a}\right\|_{T}+G_{x} x_{s, \alpha}^{a} \\
& =c\left\|x_{N}^{a}-x_{s}^{a}+(1-\alpha)\left(x_{s}^{a}-x_{r}\right)\right\|_{T} \\
& \quad+\alpha G_{x} x_{s}^{a}+(1-\alpha) G_{x} x_{r} \\
& \leq c\left\|x_{N}^{a}-x_{s}^{a}\right\|_{T}+(1-\alpha) c\left\|\left(x_{s}^{a}-x_{r}\right)\right\|_{T} \\
& \quad+(1 /(1+\xi))\left(f_{x}+\alpha \epsilon_{s}^{a}\right) \\
& \leq 2 \gamma \delta c+(1 /(1+\xi))\left(f_{x}+\alpha \epsilon_{s}^{a}\right) \leq f_{x}+\epsilon_{s, \alpha}^{a} .
\end{aligned}
$$

Conditions (14) and (15) are satisfied for $\epsilon_{s, \alpha}^{a} \geq$ $\max \left\{\alpha \epsilon_{s}^{a}, \frac{\alpha}{1+\xi} \epsilon_{s}^{a}\right\}$ and $(1-\alpha)\left\|x_{s}^{a}-x_{r}\right\|_{P} \leq \delta \leq$ $\frac{\xi}{2 \gamma c_{i}(1+\xi)} f_{x, i} \forall i=1, \ldots, p_{x}$, showing that $\epsilon_{s, \alpha}^{a}=\epsilon_{s}^{a}$ is a feasible choice for any $\delta$ and $\alpha$ satisfying the latter condition.

\section{Theorem IV.3 (Asymptotic Stability under $\kappa^{s}\left(x, z_{r}\right)$ )}

Let $\left(x_{r}, u_{r}\right) \in \mathcal{S}^{t r}$ be a reference steady-state. $x_{r}$ is asymptotically stable for the closed loop system 
$x(k+1)=A x(k)+B \kappa^{s}\left(x(k), z_{r}\right)$ with region of attraction $\mathcal{X}_{N}^{s}$.

Proof: Using Lemma IV.1 and IV.2, it can be shown that $V_{N}^{s *}\left(x, z_{r}\right)$ is a Lyapunov function by following the same arguments as in the hard constrained case presented in [26]. The proof is included in the appendix.

\section{Robust STABILITY}

In practice, model uncertainties or external disturbances cause a deviation from the nominal system dynamics in (3). There are two general approaches to deal with disturbances. In the case of linear systems and under certain assumptions on the MPC problem setup, the nominal control law offers inherent robust stability properties [19], [27] and robust stability can be guaranteed in an RPI set that depends on the considered disturbance size. Robust MPC schemes, on the other hand, take a worst-case disturbance size explicitly into account by changing the problem formulation and/or tightening the constraints, e.g. using a min-max or a tube-based MPC approach (see e.g. [1], [19], [20], [28] and the references therein).

In a hard constrained setup, both techniques have potential limitations. Using a nominal MPC scheme, the RPI set, in which stability can be guaranteed, may be prohibitively small for the considered disturbance size. Using a robust MPC scheme, the choice of the disturbance bound employed in the controller design is often conservative, since feasibility of the MPC problem may be lost if the disturbance exceeds the predefined bound.

The proposed soft constrained scheme can be used to improve the properties of both techniques. The nominal soft constrained method offers inherent robust stability properties and can thereby provide stability in a potentially much larger RPI set, since state constraints can be relaxed. It is important to note that, while stability is formally only guaranteed within the RPI set, the control law is defined everywhere in the enlarged feasible set. If constraint satisfaction should be guaranteed for a certain expected size of the disturbance, the soft constrained scheme can be combined with a robust MPC approach. While the robust method designs the problem for a certain disturbance bound, the use of the proposed soft constrained formulation ensures feasibility and stability of the MPC problem if the disturbance exceeds this bound. Conservatism in the choice of the disturbance bound for robust MPC can thereby be avoided and the system performance improved.

Robust stability under both the nominal soft constrained MPC scheme as well as the combination with a robust MPC approach is proven in the following using the framework of input-to-state stability.

\section{A. ISS of Nominal Soft Constrained MPC}

Assume that the system is subject to an additive uncertainty as given in (1). Because of the disturbance, the shifted sequence $\mathbf{u}^{\text {shift }}$ in (11) may no longer be feasible for $\mathbb{P}_{N}^{s}\left(x^{+}, z_{r}\right)$. For all $x^{+} \in \mathcal{X}_{N}^{s}$ there does, however, exist a feasible solution to $\mathbb{P}_{N}^{s}\left(x^{+}, z_{r}\right)$ and input-to-state stability can be shown in an RPI set $\mathcal{X}_{\mathcal{W}}^{s} \subset \mathcal{X}_{N}^{s}$. It is given by the maximum robust positively invariant set for the controlled uncertain system $x^{+}=A x+B \kappa^{s}\left(x, z_{r}\right)+w$ under the optimal soft constrained MPC control law in (9). We make use of the following result in order to show that the uncertain system in (1) under the nominal control law $\kappa^{s}\left(x, z_{r}\right)$ is input-to-state stable with respect to the (unspecified) disturbance set $\mathcal{W}$ in Theorem V.2.

Lemma V.1 (Continuity of $\boldsymbol{V}_{\boldsymbol{N}}^{\boldsymbol{s *}}(\boldsymbol{x})$ ) Consider problem $\mathbb{P}_{N}^{s}\left(x, z_{r}\right)$. The optimal value function $V_{N}^{s *}\left(x, z_{r}\right)$ is uniformly continuous in $x$ on $\mathcal{X}_{N}^{s}$.

Proof: Continuity follows directly from continuity and convexity of the cost function and the constraints in (7) as well as compactness of the constraint set for all $x \in \mathcal{X}_{N}^{s}$ (Theorem 4.3.3 in [29]), where the latter is provided by the fact that the terminal set is compact (Assumption III.1). Uniform continuity then follows, since $\mathcal{X}_{N}^{s}$ is compact (e.g. Proposition 5 in [20]).

Theorem V.2 (ISS under $\boldsymbol{\kappa}^{\boldsymbol{s}}\left(\boldsymbol{x}, \boldsymbol{z}_{\boldsymbol{r}}\right)$ ) Let $\left(x_{r}, u_{r}\right) \in \mathcal{S}^{\text {tr }}$ be a given reference steady-state. $x_{r}$ is ISS for the closed loop system $x(k+1)=A x(k)+B \kappa^{s}\left(x(k), z_{r}\right)+w(k)$ with respect to $w(k) \in \mathcal{W}$ with region of attraction $\mathcal{X}_{\mathcal{W}}^{s}$.

Proof: From the proof of Theorem IV.3 and Lemma V.1 it follows that $V_{N}^{s *}\left(x, z_{r}\right)$ is a uniformly continuous Lyapunov function and hence there exists a $\mathcal{K}$-class function $\gamma(\cdot)$, such that $\left|V_{N}^{s *}\left(y, z_{r}\right)-V_{N}^{s *}\left(x, z_{r}\right)\right| \leq \gamma(\|y-x\|)$ (see, e.g., [20], A.11) as well as a $\mathcal{K}_{\infty}$-class function $\alpha_{3}(\cdot)$ such that $V_{N}^{s *}\left(A x+B \kappa^{s}\left(x, z_{r}\right), z_{r}\right)-V_{N}^{s *}\left(x, z_{r}\right) \leq-\alpha_{3}\left(\left\|x-x_{r}\right\|\right)$. It follows from these facts that

$$
\begin{aligned}
& V_{N}^{s *}\left(x^{+}, z_{r}\right)-V_{N}^{s *}\left(x, z_{r}\right) \\
& =V_{N}^{s *}\left(A x+B \kappa^{s}\left(x, z_{r}\right)+w, z_{r}\right)-V_{N}^{s *}\left(x, z_{r}\right) \\
& \quad+V_{N}^{s *}\left(A x+B \kappa^{s}\left(x, z_{r}\right), z_{r}\right)-V_{N}^{s *}\left(A x+B \kappa^{s}\left(x, z_{r}\right), z_{r}\right) \\
& \leq-\alpha_{3}\left(\left\|x-x_{r}\right\|\right)+\gamma(\|w\|),
\end{aligned}
$$

i.e. $V_{N}^{s *}\left(x, z_{r}\right)$ is an ISS-Lyapunov function with respect to $w \in \mathcal{W}$ and $x_{r}$ is ISS for the closed-loop system.

The uncertain system controlled by the soft constrained control law $\kappa^{s}\left(x, z_{r}\right)$ is hence input-to-state stable against sufficiently small disturbances. Since the RPI set $\mathcal{X}_{\mathcal{W}}^{s}$ depends on $\mathcal{W}$, the size of the disturbances and the corresponding region, for which stability can be formally guaranteed, depend on the particular system of interest.

In the following section we will show that the previously presented results can be directly extended to the combination of a robust and soft constrained MPC framework, in order to take advantage of both properties.

\section{B. Combination of Robust and Soft Constrained MPC}

We assume in the following that the system is affected by two uncertainties:

$$
x(k+1)=A x(k)+B u(k)+w_{1}(k)+w_{2}(k),
$$

where $w_{1} \in \mathcal{W}_{1}, w_{2} \in \mathcal{W}_{2}$ and $\mathcal{W}_{1}, \mathcal{W}_{2}$ are convex and compact sets that each contain the origin. The disturbance $w_{1}$ is explicitly taken into account by using a robust MPC 
technique, which provides constraint satisfaction and stability in the presence of $w_{1}$. Feasibility and stability in the presence of $w_{2}$ is guaranteed by means of the proposed soft constrained scheme.

In this work, we apply the tube-based robust MPC approach for linear systems [28]. The method is based on the use of a feedback policy of the form $u=\bar{u}+K(x-\bar{x})$ that bounds the effect of the disturbance $w_{1}$ and keeps the states $x$ of the uncertain system under $w_{1}$ close to the states of the nominal system in (3). The use of tightened state and input constraints ensures feasibility of the uncertain system in (16) despite the disturbance $w_{1}: \overline{\mathbb{X}}=\mathbb{X} \ominus \mathcal{Z} \triangleq\left\{x \mid G_{x} x \leq \bar{f}_{x}\right\}, \overline{\mathbb{U}}=$ $\mathbb{U} \ominus K \mathcal{Z} \triangleq\left\{u \mid G_{u} u \leq \bar{f}_{u}\right\}$, with $\bar{f}_{x, i}=f_{x, i}-h_{\mathcal{Z}}\left(G_{x, i}^{T}\right), i=$ $1, \ldots, p_{x}, \bar{f}_{u, i}=f_{u, i}-h_{\mathcal{Z}}\left(K^{T} G_{u, i}^{T}\right), i=1, \ldots, p_{u}$, where $\mathcal{Z}$ is an RPI set for the controlled system $x^{+}=(A+B K) x+w_{1}$ and $h_{\mathcal{Z}}(a)=\sup _{x \in \mathcal{Z}} a^{T} x$ is the support function of $\mathcal{Z}$ evaluated at $a$. Note that the tightened state and input constraints again result in compact polytopes. See [28] for a detailed description of the method. The tracking approach described in Section II was extended to a robust tube-based MPC method for tracking in [23], which will be combined in the following with the soft constrained scheme proposed in Section III.

Problem $\mathbb{P}_{N}^{r s}\left(x, z_{r}\right) \quad$ (Robust soft constrained MPC problem)

$$
\begin{aligned}
V_{N}^{r s *}\left(x, z_{r}\right)=\min _{\overline{\mathbf{x}}, \overline{\mathbf{u}}, \bar{z}_{s}, \bar{\epsilon}, z_{r}} & V_{N}^{s}\left(\overline{\mathbf{x}}, \overline{\mathbf{u}}, \bar{z}_{s}, \overline{\boldsymbol{\epsilon}}, z_{r}\right)+V_{f}\left(x-\bar{x}_{0}\right) \\
\text { s.t. } \quad & x \in \bar{x}_{0} \oplus \mathcal{Z}, \\
& (7 \mathrm{c})-(7 \mathbf{j})
\end{aligned}
$$

where in the constraints (7c)-(7j), $f_{x}, f_{u}$ and $\mathcal{E}_{f}^{s}\left(x_{s}, u_{s}\right)$ are replaced with $\bar{f}_{x}, \bar{f}_{u}$ and $\overline{\mathcal{E}}_{f}^{s}\left(\bar{x}_{s}, \bar{u}_{s}\right)$, respectively. The conditions on the robust terminal set for tracking $\overline{\mathcal{E}}_{f}^{s}\left(\bar{x}_{s}, \bar{u}_{s}\right)$ are obtained by replacing the input constraints $\mathbb{U}$ in Assumption III.1 with $\overline{\mathbb{U}}$. Compared to [28], we propose to augment the cost with the term $V_{f}\left(x-\bar{x}_{0}\right)$, which offers the advantage of directly providing an ISS Lyapunov function (Theorem V.4, see also [26], [30] for more details).

Remark V.3. The use of tightened state constraints in the soft constrained formulation, i.e. replacing $f_{x}$ with $\bar{f}_{x}$, has the advantage that the behavior of the robust MPC controller is recovered if the constraints can be enforced. Stability would, however, also be provided by using $f_{x}$.

The set of feasible initial states of the robust soft constrained problem is denoted by $\mathcal{X}_{N}^{r s}$. The robust formulation does not change the problem structure and Problem $\mathbb{P}_{N}^{r s}\left(x, z_{r}\right)$ again results in a convex SOCP. For a given state $x \in \mathcal{X}_{N}^{r s}$, the solution of $\mathbb{P}_{N}^{r s}\left(x, z_{r}\right)$ yields the optimal control sequence $\overline{\mathbf{u}}^{r s *}\left(x, z_{r}\right)$ and the optimal first tube center $\bar{x}_{0}^{r s *}\left(x, z_{r}\right)$. The robust soft constrained control law is then given in a receding horizon fashion by

$$
\kappa^{r s}\left(x, z_{r}\right) \triangleq \bar{u}_{0}^{r s *}\left(x, z_{r}\right)+K\left(x-\bar{x}_{0}^{r s *}\left(x, z_{r}\right)\right) .
$$

Input-to-state stability will in the following be shown for the robust invariant set $\mathcal{X}_{\mathcal{W}}^{r s} \subseteq \mathcal{X}_{N}^{r s}$, given by the maximum robust positively invariant set for the controlled uncertain system $x^{+}=A x+B \kappa^{r s}\left(x, z_{r}\right)+w_{1}+w_{2}$ with $w_{1} \in \mathcal{W}_{1}, w_{2} \in \mathcal{W}_{2}$.
Let $\overline{\mathcal{S}}^{t r} \triangleq\left\{\left(x_{s}, u_{s}\right) \in \mathcal{S}^{t r} \mid(1+\xi) G_{x} x_{s} \leq \bar{f}_{x},(1+\xi) G_{u} u_{s} \leq\right.$ $\left.\bar{f}_{u}\right\}$.

Theorem V.4 (ISS under $\boldsymbol{\kappa}^{r s}\left(\boldsymbol{x}, \boldsymbol{z}_{r}\right)$ ) Let $\left(x_{r}, u_{r}\right) \in \overline{\mathcal{S}}^{\text {tr }}$ be a given reference steady-state. $x_{r}$ is ISS for the closed loop system $x(k+1)=A x(k)+B \kappa^{r s}\left(x(k), z_{r}\right)+w_{1}(k)+w_{2}(k)$ with respect to $w_{1}(k) \in \mathcal{W}_{1}$ and $w_{2}(k) \in \mathcal{W}_{2}$ with region of attraction $\mathcal{X}_{\mathcal{W}}^{r s}$.

Proof: The first part of the proof assumes $w_{2}=0$ and follows similar steps as in Section IV to show that $V_{N}^{r s *}\left(x, z_{r}\right)$ is an ISS Lyapunov function with respect to $w_{1}$. A more detailed version of this part of the proof can also be found in [26]. The second part of the proof then shows that $V_{N}^{r s *}\left(x, z_{r}\right)$ is also an ISS Lyapunov function with respect to $w_{2}$. In the following, we omit the dependence of the optimal solution on $\left(x, z_{r}\right)$. Let $w_{2}=0$, i.e. $x^{+}=A x+B \kappa^{r s}\left(x, z_{r}\right)+w_{1}$. Lemma IV.1 extends to the robust problem setup showing feasibility of the shifted sequence with $\overline{\boldsymbol{\epsilon}}^{\text {shift }}=\left[\bar{\epsilon}_{1}^{r s *}, \ldots, \bar{\epsilon}_{N-1}^{r s *}, 0, \bar{\epsilon}_{s}^{r s *}\right]$. Using uniform continuity of $V_{f}(\cdot), x^{+}=\bar{x}_{1}^{r s *}+(A+B K)\left(x-\bar{x}_{0}^{r s *}\right)+w_{1}$ and Assumption II.5, it can be shown that there exists a $\mathcal{K}$-class function $\gamma_{1}(\cdot)$ such that $V_{f}\left(x^{+}-\bar{x}_{1}^{r s *}\right)-V_{f}\left(x-\bar{x}_{0}^{r s *}\right) \leq$ $-\left\|x-\bar{x}_{0}^{r s *}\right\|_{Q}^{2}+\gamma_{1}\left(\left\|w_{1}\right\|\right)$. Using standard arguments and convexity of $\|\cdot\|_{Q}^{2}$, it then follows that

$$
\begin{aligned}
& V_{N}^{r s *}\left(A x+B \kappa^{r s}\left(x, z_{r}\right)+w_{1}, z_{r}\right)-V_{N}^{r s *}\left(x, z_{r}\right) \\
& \leq V_{N}^{s}\left(\overline{\mathbf{x}}^{\text {shift }}, \overline{\mathbf{u}}^{\text {shift }}, \bar{z}_{s}^{r *}, \overline{\boldsymbol{\epsilon}}^{\text {shift }}, z_{r}\right)+V_{f}\left(x^{+}-\bar{x}_{1}^{r s *}\right) \\
& \quad-V_{N}^{s}\left(\overline{\mathbf{x}}^{r s *}, \overline{\mathbf{u}}^{r s *}, \bar{z}_{s}^{r s *}, \overline{\boldsymbol{\epsilon}}^{r s *}, z_{r}\right)-V_{f}\left(x-\bar{x}_{0}^{r s *}\right) \\
& \leq-\left\|\bar{x}_{0}^{r s *}-\bar{x}_{s}^{r s *}\right\|_{Q}^{2}-\left\|x-\bar{x}_{0}^{r s *}\right\|_{Q}^{2}+\gamma_{1}\left(\left\|w_{1}\right\|\right) \\
& \leq-\frac{1}{2}\left\|x-\bar{x}_{s}^{r s *}\right\|_{Q}^{2}+\gamma_{1}\left(\left\|w_{1}\right\|\right) .
\end{aligned}
$$

Following similar arguments as in the proof of Theorem IV.3 and using optimality of $V_{N}^{r s *}\left(x, z_{r}\right)$ it can be shown that there exist $\mathcal{K}_{\infty}$-class functions $\underline{\alpha}(\cdot), \bar{\alpha}(\cdot)$ such that $V_{N}^{r s *}\left(x, z_{r}\right) \geq$ $\underline{\alpha}\left(\left\|x-x_{r}\right\|\right) \forall x \in \mathcal{X}_{N}^{r s}$ and $V_{N}^{r s *}\left(x, z_{r}\right) \leq \bar{\alpha}\left(\left\|x-x_{r}\right\|\right) \forall x \in$ $\overline{\mathcal{E}}_{f}^{s}\left(x_{r}, u_{r}\right) \oplus \mathcal{Z}$. The condition that remains to be shown is that (18) also provides a strict decrease that is a function of $\left\|x-x_{r}\right\|_{Q}^{2}$. Lemma IV.2 directly extends to the robust problem formulation by replacing the constraints with the tightened form (Part 1)) and since the additional cost term $V_{f}\left(x-\bar{x}_{0}\right)$ does not contain the steady-state $x_{s}$ and is irrelevant for the argument (Part 2)). Considering the same cases and arguments as in the proof of Theorem IV.3, it can then be shown that there exists a $\mathcal{K}_{\infty}$-class function $\alpha_{3}(\cdot)$ such that

$$
\begin{aligned}
& V_{N}^{r s *}\left(A x+B \kappa^{r s}\left(x, z_{r}\right)+w_{1}, z_{r}\right)-V_{N}^{r s *}\left(x, z_{r}\right) \\
& \leq-\alpha_{3}\left(\left\|x-x_{r}\right\|\right)+\gamma_{1}\left(\left\|w_{1}\right\|\right) .
\end{aligned}
$$

For the second part of the proof, uniform continuity of the optimal value function $V_{N}^{r s *}\left(x, z_{r}\right)$ follows as in the non-robust case from the proof of Lemma V.1. Therefore, there exists a $\mathcal{K}$-class function $\gamma_{2}(\cdot)$, such that $\left|V_{N}^{r s *}(y)-V_{N}^{r s *}(x)\right| \leq$ $\gamma_{2}(\|y-x\|)$ and we obtain

$$
\begin{aligned}
& V_{N}^{r s *}\left(A x+B \kappa^{r s}\left(x, z_{r}\right)+w_{1}+w_{2}, z_{r}\right)-V_{N}^{r s *}\left(x, z_{r}\right) \\
& \leq-\alpha_{3}\left(\left\|x-x_{r}\right\|\right)+\gamma_{1}\left(\left\|w_{1}\right\|\right)+\gamma_{2}\left(\left\|w_{2}\right\|\right),
\end{aligned}
$$

proving the result.

Theorem V.4 proves ISS of the uncertain system in (16) controlled by $\kappa^{r s}\left(x, z_{r}\right)$ in (17) with respect to the disturbances 
$w_{1} \in \mathcal{W}_{1}$ and $w_{2} \in \mathcal{W}_{2}$ and shows that the results presented for the soft constrained MPC method can directly be extended to the combination of a robust and soft constrained approach.

\section{NUMERICAL EXAMPLES}

In this section, the proposed methods for soft constrained and robust soft constrained MPC are illustrated for a smallscale example and computation times for a large-scale problem are provided. All set computations were carried out using YALMIP [31] and the MPT toolbox [32].

\section{A. Illustrative Example}

Consider the following unstable system:

$$
x(k+1)=\left[\begin{array}{cc}
1.05 & 1 \\
0 & 1
\end{array}\right] x(k)+\left[\begin{array}{c}
1 \\
0.5
\end{array}\right] u(k)+w(k) .
$$

The prediction horizon was chosen to be $N=5$, the constraints on the states and control inputs to $\|x\|_{\infty} \leq 5$ and $\|u\|_{\infty} \leq 1, Q=I, R=1$ and $S=100 I$. The terminal cost function $V_{f}(\cdot)$ is taken as the unconstrained infinite horizon optimal value function for the nominal system with $P=\left[\begin{array}{ll}1.9119 & 0.2499 \\ 0.2499 & 2.6510\end{array}\right]$ and $\kappa_{f}(x)=K\left(x-x_{s}\right)+u_{s}$ is the corresponding optimal LQR controller. The exact penalty multipliers were chosen as $\rho_{\epsilon}=\rho_{x}=\rho_{u}=100$, which was observed to provide optimality in $\mathcal{X}_{N}$. For simplicity, we take $x_{r}=0, u_{r}=0$ as the reference steady-state for the following illustrations.

1) Soft Constrained MPC: The feasible set $\mathcal{X}_{5}^{s}$ and the enlarged terminal set $\mathcal{C}_{f}^{s}$ for the soft constrained approach $\mathbb{P}_{N}^{s}\left(x, z_{r}\right)$ are illustrated and compared with the feasible set $\mathcal{X}_{5}$ and terminal set $\mathcal{C}_{f}$ for the hard constrained problem $\mathbb{P}_{N}\left(x, z_{r}\right)$ in Figure 2, which demonstrates that the soft constrained approach significantly enlarges the feasible set and thereby the region of attraction for the nominal closed-loop system. This shows that the proposed method provides the benefits of soft constraints and ensures feasibility of the optimization problem in a large region while still guaranteeing stability of the closed-loop system.

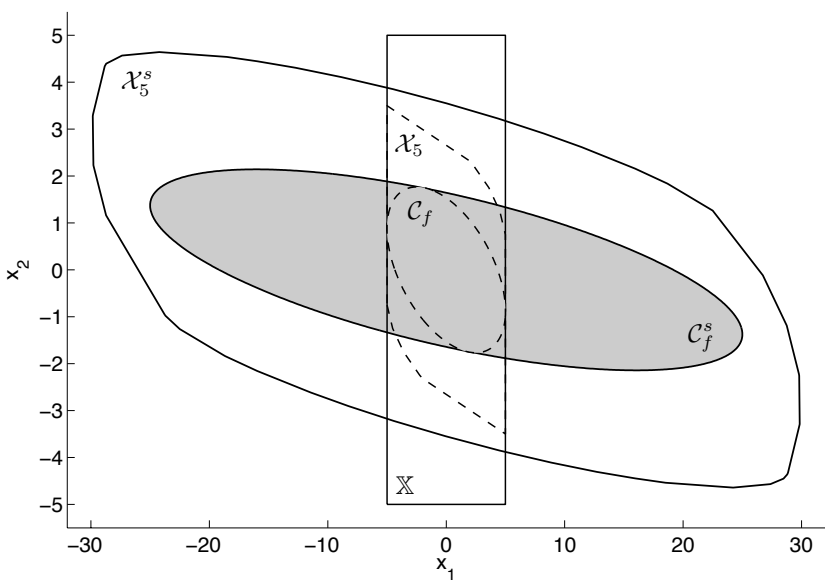

Fig. 2. Feasible and terminal set for the soft constrained problem $\mathbb{P}_{N}^{s}\left(x, z_{r}\right)$ for $N=5$ in comparison with the feasible and terminal set of the hard constrained problem $\mathbb{P}_{N}\left(x, z_{r}\right)$.

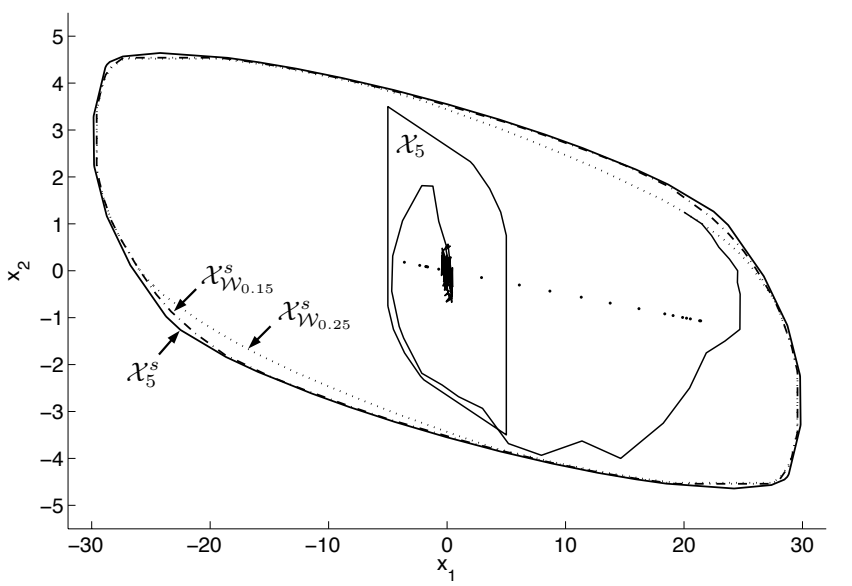

Fig. 3. Feasible set and RPI sets of $\mathbb{P}_{N}^{s}\left(x, z_{r}\right)$ for $\bar{w} \in\{0.15,0.25\}$ together with a closed-loop trajectory starting at $x(0)=[20,1.25]^{T}$ under a sequence of extreme disturbances. Dots represent the optimal steady-state $x_{s}^{s *}(x(k), 0)$ at each sampling time.

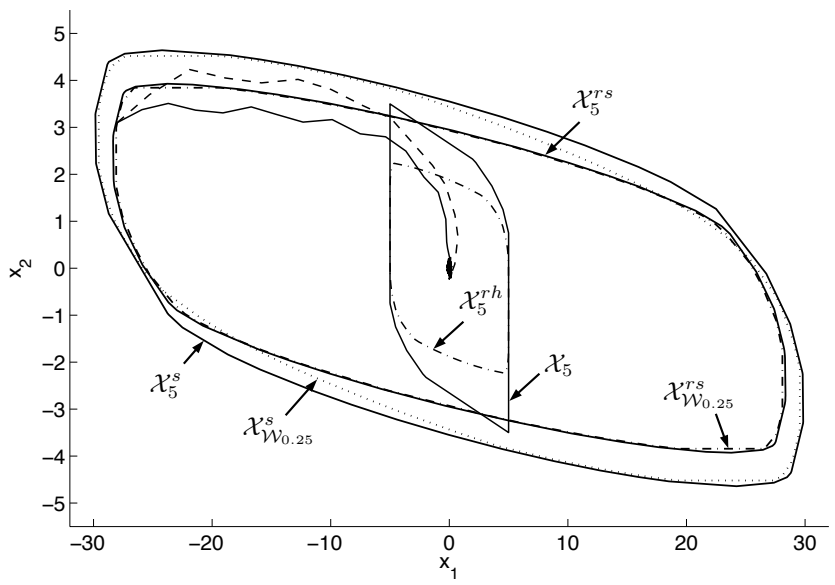

Fig. 4. Feasible set and RPI sets of $\mathbb{P}_{N}^{s}\left(x, z_{r}\right)$ and $\mathbb{P}_{N}^{r s}\left(x, z_{r}\right)$ for $\bar{w}_{1}=0.1, \bar{w}_{2}=0.15$ together with closed-loop trajectories starting at $x(0)=\left[\begin{array}{lll}-28 & 3.1\end{array}\right]^{T}$, where the dashed line solves $\mathbb{P}_{N}^{s}(x, 0)$ and the solid line $\mathbb{P}_{N}^{r s}(x, 0) . \mathcal{X}_{5}^{r h}$ is the feasible set for the robust hard constrained problem.

We now analyze the robust stability properties of the example system (19) under the soft constrained control law $\kappa^{s}\left(x, z_{r}\right)$ in (9). Figure 3 shows the size of the RPI sets $\mathcal{X}_{\mathcal{W}_{\bar{w}}}^{s}$ for two bounds $\mathcal{W}_{\bar{w}} \triangleq\left\{w \mid\|w\|_{\infty} \leq \bar{w}\right\}$, with $\bar{w} \in\{0.15,0.25\}$ assessed by sampling. Note that for a robust tube-based approach the feasible set is always a subset of $\mathcal{X}_{N}$. This demonstrates the advantage of the soft constrained approach, where input-to-state stability in the presence of a comparably large disturbance $w \in \mathcal{W}_{0.25}$ can be guaranteed in the RPI set $\mathcal{X}_{\mathcal{W}_{0.25}} \supset \mathcal{X}_{N}$. In addition, a closed-loop trajectory starting at $x(0)=\left[\begin{array}{ll}20 & 1.25\end{array}\right]^{T}$ under a sequence of extreme disturbances with $\|w(k)\|_{2}=0.25 \forall k \geq 0$ is shown as well as the corresponding optimal steady-states at each sampling time, demonstrating that the closed-loop system is stable and does not leave the RPI set $\mathcal{X}_{\mathcal{W}_{0.25}}$.

2) Robust Soft Constrained MPC: In the following, the properties of the robust soft constrained MPC approach described in Section V-B are illustrated. Consider again system (19) with $w(k)=w_{1}(k)+w_{2}(k)$ that is now subject to two types of disturbances. Figure 4 shows the comparison of the feasible set $\mathcal{X}_{5}^{r s}$ and the RPI set $\mathcal{X}_{\mathcal{W}_{0.25}}^{r s}$ for $w_{1} \in$ 
$\mathcal{W}_{\bar{w}_{1}}, w_{2} \in \mathcal{W}_{\bar{w}_{2}}$ with $\bar{w}_{1}=0.1, \bar{w}_{2}=0.15$ in comparison with the feasible set $\mathcal{X}_{5}^{s}$ and the RPI set $\mathcal{X}_{\mathcal{W}_{0.25}}^{s}$ of the pure soft constrained approach. The feasible set of the hard constrained robust MPC problem, i.e. Problem $\mathbb{P}_{N}^{r s}\left(x, z_{r}\right)$ with $\left(x_{s}, u_{s}\right) \in$ $\mathcal{S}, \epsilon_{s}=0, \epsilon_{i}=0, w=w_{1}+w_{2} \in \mathcal{W}_{0.25}$ is denoted by $\mathcal{X}_{5}^{r h}$. Due to the tightening of the input constraints, the robust soft constrained approach has a smaller feasible set when compared to the pure soft constrained method. However, in comparison with the hard constrained robust MPC method, the feasible set for the combined approach is significantly larger, while still guaranteeing ISS with respect to $w_{1}$ in $\mathcal{X}_{5}^{r s}$. The

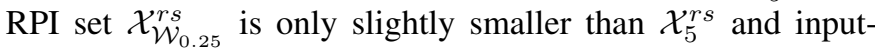
to-state stability with respect to the combined disturbance $w=w_{1}+w_{2} \in \mathcal{W}_{0.25}$ is provided in a comparably large set. Closed-loop trajectories starting from $x(0)=\left[\begin{array}{lll}-28 & 3.1\end{array}\right]^{T}$ are shown for both the robust soft constrained and the pure soft constrained approach under a sequence of extreme disturbances $\left\|w_{1}(k)\right\|_{2}=0.1$ and a disturbance $\left\|w_{2}(k)\right\|_{2} \in \mathcal{W}_{0.15}$ $\forall k \geq 0$ that additionally affects the system at every third sampling time. Figure 4 demonstrates that both approaches provide input-to-state stability of the closed-loop system. The robust soft constrained approach steers the system earlier towards the origin and the trajectory remains in $\mathcal{X}_{\mathcal{W}_{0.25}}^{r s}$, since the robust formulation is designed to counteract the disturbance $w_{1}$. The soft constrained approach allows for a larger deviation of the state within the RPI set $\mathcal{X}_{\mathcal{W}_{0.25}}^{s}$. This shows that by using a combination of a robust and soft constrained method, robustness against a certain disturbance size can be provided while ensuring stability if the disturbance exceeds this bound.

\section{B. Large-Scale Example}

The following example demonstrates that the proposed soft constrained MPC approach can be applied to problems of significant size. We consider two systems of 6 and 12 oscillating masses, which are interconnected by springs and dampers and are connected to walls on each side. The actuators exert tension between different masses. The system with 12 masses is illustrated in Fig. 5, where the six masses problem is obtained by considering the first six masses from the left. The

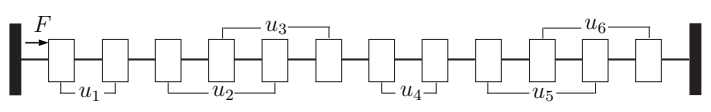

Fig. 5. System of 12 oscillating masses.

masses have value $1 \mathrm{~kg}$, the spring constant is $k=0.7 \mathrm{~N} / \mathrm{m}$, the damping constant is $0.1 \mathrm{Ns} / \mathrm{m}$ and an external force $F=0.5 x_{1}$ is applied to the first mass on the left. The control inputs are constrained to lie in $\pm 1 \mathrm{~N}$ and the displacement of the masses is constrained in $\pm 4 \mathrm{~m}$. The system is discretized with sampling time $t_{s}=0.5 \mathrm{~s}$. The parameters of the problem $\mathbb{P}_{N}^{s}\left(x, z_{r}\right)$ are $N=10, Q=I$ and $R=I, S=100 I$, $\rho_{\epsilon}=\rho_{x}=\rho_{u}=100$.

In order to exemplify the computation times that can be achieved for the proposed soft constrained procedure, the resulting SOCP was solved using the solver MOSEK [12] and ECOS [13], which is particularly designed to run on embedded platforms, on a MacBook Pro with Intel Core i7 CPU at 2.6 $\mathrm{GHz}$. The problem dimensions, the size of the resulting SOCP and the minimum, average and maximum computation times for the 6 and 12 masses problem using 100 randomly sampled initial states are given in Table I. The results show that the SOCP can be solved in the millisecond range even for big problem dimensions, demonstrating the practical applicability of the method.

\section{CONCLUSIONS}

In this paper, a new soft constrained MPC method based on a finite horizon MPC scheme for tracking was introduced that provides closed-loop stability even for unstable systems. The approach combines the benefits of a soft constrained scheme with the desirable properties of MPC and ensures feasibility of the optimization in a large region of the state space while guaranteeing closed-loop stability. The proposed control law preserves the optimal tracking performance of the corresponding hard constrained MPC approach whenever the state constraints can be enforced. Asymptotic stability of all feasible reference steady-states for the nominal system under the soft constrained control law was shown, as well as inputto-state stability in the presence of additive disturbances. The proposed soft constrained formulation was combined with a robust approach, in order to provide constraint satisfaction despite a certain disturbance size, while ensuring feasibility and stability for exceeding disturbances by means of soft constraints. It was shown that the results on input-to-state stability extend to this case. The presented numerical examples illustrate the properties of the new soft constrained scheme and demonstrate that it can be applied to problems of significant size in order to ensure safety and feasibility during online operation.

\section{ACKNOWLEDGMENT}

The authors thank A. Domahidi for his valuable comments and support with the implementation in ECOS. The research leading to these results was supported by the European Union Seventh Framework Programme FP7/2007-2013 under grant agreement number FP7-ICT- 2009-4 248940.

\section{APPENDIX}

Detailed proofs of Lemma III.2, result 2) in Lemma IV.2 and Theorem IV.3 are provided in the following, where the last two are similar to the proofs of Lemma 5.6 and 5.7 in [26], but are included to make the paper self-contained.

\section{Proof of Lemma III.2:}

Consider a parametrization of the steady-state by the parameter $\theta \in \mathbb{R}^{n_{\theta}}[9]:$

$$
\left[\begin{array}{l}
x_{s} \\
u_{s}
\end{array}\right]=M \theta=\left[\begin{array}{l}
M_{x} \\
M_{u}
\end{array}\right] \theta,
$$

where the columns of $M$ form a basis for the null space of the matrix $\left[\begin{array}{ll}I-A & -B\end{array}\right], n_{\theta}$ is the dimension of the null space and $M_{x}, M_{u}$ are appropriate partitions of $M$. We define the augmented system $v^{T}=\left[\begin{array}{ll}x^{T}-x_{s}^{T} & \theta^{T}\end{array}\right]$ with dynamics $v^{+}=$ 


\begin{tabular}{|c|c|c|c|c|c|c|c|c|}
\hline M & $n$ & $m$ & $N$ & nvar & neq & ineq & $\begin{array}{c}\text { ECOS [13] } \\
(\min / \text { avg / } \max )\end{array}$ & $\begin{array}{c}\text { MOSEK [12] } \\
\text { (min / avg / max) }\end{array}$ \\
\hline 6 & 12 & 3 & 10 & 207 & 132 & 900 & $16.7 / 20.2 / 25.0 \mathrm{~ms}$ & $10.8 / 13.0 / 18.9 \mathrm{~ms}$ \\
\hline 12 & 24 & 6 & 10 & 372 & 264 & 2295 & $93.3 / 106.6 / 154.1 \mathrm{~ms}$ & $49.3 / 57.3 / 72.9 \mathrm{~ms}$ \\
\hline
\end{tabular}

TABLE I

COMPUTATION TIMES FOR SOLVING THE SOFT CONSTRAINED PROBLEM $\mathbb{P}_{N}^{s}\left(x, z_{r}\right)$ FOR 100 RANDOMLY SAMPLED INITIAL STATES. NVAR, NEQ AND INEQ DENOTE THE NUMBER OF VARIABLES, EQUALITY CONSTRAINTS AND INEQUALITY CONSTRAINTS, RESPECTIVELY, IN THE RESULTING SOCP.

$A_{v, K} v$, where $A_{v, K}=\left[\begin{array}{cc}A+B K & 0 \\ 0 & I\end{array}\right]$. An ellipsoidal invariant set of the form

$$
\Omega_{f} \triangleq\left\{v \in \mathbb{R}^{n} \mid v^{T}\left[\begin{array}{cc}
Q_{1}^{-1} & \\
& Q_{2}^{-1}
\end{array}\right] v \leq 1\right\}
$$

can be computed by solving a convex LMI [10], where only the input constraints are considered and the state constraints are neglected:

$$
\begin{aligned}
& {\left[\begin{array}{ll}
Q_{1} & \\
& Q_{2}
\end{array}\right]=\underset{Q_{1}, Q_{2}}{\operatorname{argmin}}\left\{-\log \operatorname{det}\left[\begin{array}{ll}
Q_{1} & \\
& Q_{2}
\end{array}\right] \mid\left[\begin{array}{cc}
Q_{1} & Q_{1} A_{K}^{T} \\
A_{K} Q_{1} & Q_{1}
\end{array}\right] \succeq 0,\right.} \\
& \left.\left\|Q_{1}^{\frac{1}{2}} K^{T} G_{u, j}^{T}\right\|_{2}^{2}+\left\|Q_{2}^{\frac{1}{2}} M_{u}^{T} G_{u, j}^{T}\right\|_{2}^{2} \leq f_{u, j}^{2} \forall j=1, \ldots, p_{u}\right\},
\end{aligned}
$$

with $A_{K}=A+B K$. Using the relationship $\theta=M_{x}^{T} x_{s}+$ $M_{u}^{T} u_{s}$ this can be directly transformed into the ellipsoidal terminal set $\mathcal{E}_{f}^{s}\left(x_{s}, u_{s}\right)$ in (8) with $T=Q_{1}^{-1}$ and $r\left(x_{s}, u_{s}\right)=$ $\left(M_{x}^{T} x_{s}+M_{u}^{T} u_{s}\right)^{T} Q_{2}^{-1}\left(M_{x}^{T} x_{s}+M_{u}^{T} u_{s}\right)$. Note that if problem (21) is unbounded, boundedness can be imposed by setting a large upper bound on $x_{s}$, e.g. $G_{x} M_{x} \theta \leq \gamma f_{x}$ for some large constant $\gamma>0$.

\section{Proof of result 2) in Lemma IV.2:}

We denote $A_{K} \triangleq A+B K, \Delta x_{0}^{a} \triangleq x_{0}^{a}-x_{s}^{a}, \Delta x_{s}^{a} \triangleq x_{s}^{a}-x_{r}$, $\Delta u_{s}^{a} \triangleq u_{s}^{a}-u_{r}, \nu=1-\alpha$. By Assumption II.5, $V_{f}(x)$ is a Lyapunov function and $P \succeq Q+K^{T} R K$, therefore $\left\|A_{K}^{i} x\right\|_{Q} \leq\left\|A_{K}^{i} x\right\|_{P} \leq\|x\|_{P}$ and $\left\|A_{K}^{i} x\right\|_{K^{T} R K} \leq$ $\left\|A_{K}^{i} x\right\|_{P} \leq\|x\|_{P}$ From the use of the auxiliary control law, i.e. $u_{i}^{a}=K A_{K}^{i} \Delta x_{0}^{a}+u_{s}^{a}$, and recalling that $x_{s}^{a}=A x_{s}^{a}+B u_{s}^{a}$ we obtain

$$
\begin{aligned}
& l\left(x_{i}^{a}-x_{s, \alpha}^{a}, u_{i}^{a}-u_{s, \alpha}^{a}\right)-l\left(x_{i}^{a}-x_{s}^{a}, u_{i}^{a}-u_{s}^{a}\right) \\
& =\left\|A_{K}^{i} \Delta x_{0}^{a}+\nu \Delta x_{s}^{a}\right\|_{Q}^{2}+\left\|K A_{K}^{i} \Delta x_{0}^{a}+\nu \Delta u_{s}^{a}\right\|_{R}^{2} \\
& \quad-\left\|A_{K}^{i} \Delta x_{0}^{a}\right\|_{Q}^{2}-\left\|K A_{K}^{i} \Delta x_{0}^{a}\right\|_{R}^{2}, \\
& \leq 2 \nu\left\|A_{K}^{i} \Delta x_{0}^{a}\right\|_{Q}\left\|\Delta x_{s}^{a}\right\|_{Q}+\nu^{2}\left\|\Delta x_{s}^{a}\right\|_{Q}^{2} \\
& \quad+2 \nu\left\|A_{K}^{i} \Delta x_{0}^{a}\right\|_{K^{T} R K}\left\|\Delta u_{s}^{a}\right\|_{R}+\nu^{2}\left\|\Delta u_{s}^{a}\right\|_{R}^{2} \\
& \leq 3 \nu^{2}\left\|\Delta x_{s}^{a}\right\|_{P}^{2}+2 \nu^{2}\left\|\Delta x_{s}^{a}\right\|_{P}\left\|\Delta u_{s}^{a}\right\|_{R}+\nu^{2}\left\|\Delta u_{s}^{a}\right\|_{R}^{2}
\end{aligned}
$$

and similarly $V_{f}\left(x_{N}^{a}-x_{s, \alpha}^{a}\right)-V_{f}\left(x_{N}^{a}-x_{s}^{a}\right) \leq 3 \nu^{2}\left\|\Delta x_{s}^{a}\right\|_{P}^{2}$. Using $V_{o}\left(x_{s, \alpha}^{a}-x_{r}, u_{s, \alpha}^{a}-u_{r}\right)=\alpha V_{o}\left(\Delta x_{s}^{a}, \Delta u_{s}^{a}\right)$, we get

$$
\begin{aligned}
& V_{N}\left(\mathbf{x}^{a}, \mathbf{u}^{a}, z_{s, \alpha}^{a}, z_{r}\right)-V_{N}\left(\mathbf{x}^{a}, \mathbf{u}^{a}, z_{s}^{a}, z_{r}\right)+\nu^{2}\left\|\Delta x_{s}^{a}\right\|_{P}^{2} \\
& \leq \nu\left[3(N+1)\left\|\Delta x_{s}^{a}\right\|_{P}^{2} \nu+2 N\left\|\Delta x_{s}^{a}\right\|_{P}\left\|\Delta u_{s}^{a}\right\|_{R} \nu\right. \\
& \left.\quad+N\left\|\Delta u_{s}^{a}\right\|_{R}^{2} \nu-V_{o}\left(\Delta x_{s}^{a}, \Delta u_{s}^{a}\right)\right] \leq 0,
\end{aligned}
$$

which is satisfied for

$0<\nu \leq \frac{V_{o}\left(\Delta x_{s}^{a}, \Delta u_{s}^{a}\right)}{3(N+1)\left\|\Delta x_{s}^{a}\right\|_{P}^{2}+2 N\left\|\Delta x_{s}^{a}\right\|_{P}\left\|\Delta u_{s}^{a}\right\|_{R}+N\left\|\Delta u_{s}^{a}\right\|_{R}^{2}}$ and hence $\alpha<1$, proving the result.

\section{Proof of Theorem IV.3:}

We will show that $V_{N}^{s *}\left(x, z_{r}\right)$ is a Lyapunov function. Let $\left(x_{s}^{s *}, u_{s}^{s *}\right)$ be the optimal steady-state for state $x$ and reference $z_{r}$. Feasibility of $\mathbb{P}_{N}^{s}\left(x^{+}, z_{r}\right)$ follows from feasibility of the shifted sequence shown in Lemma IV.1. We have that $V_{N}^{s *}\left(x, z_{r}\right) \geq\left\|x-x_{s}^{*}\right\|_{Q}^{2}+V_{o}\left(x_{s}^{*}-x_{r}\right) \geq \underline{\alpha}\left(\left\|x-x_{r}\right\|\right) \forall x \in$ $\mathcal{X}_{N}^{s}$. By optimality of $V_{N}^{s *}\left(x, z_{r}\right)$ we obtain $V_{N}^{s *}\left(x, z_{r}\right) \leq$ $V_{f}\left(x-x_{r}\right) \leq \bar{\alpha}\left(\left\|x-x_{r}\right\|\right) \forall x \in \mathcal{E}_{f}^{s}\left(x_{r}, u_{r}\right) \cap \mathbb{X}$, where $\underline{\alpha}(\cdot), \bar{\alpha}(\cdot)$ are suitable $\mathcal{K}_{\infty}$-class functions. The important condition to prove is that $\Delta V=V_{N}^{s *}\left(x^{+}, z_{r}\right)-V_{N}^{s *}\left(x, z_{r}\right) \leq$ $-\beta\left\|x-x_{r}\right\|_{Q}^{2}$ with $\beta>0$, which will be shown by considering the following two cases. If the state $x^{+}$is sufficiently far away from the optimal artificial steady-state at the previous time step $x_{s}^{s *}$, then the decrease obtained by the shifted sequence when keeping the same artificial steady-state provides $\beta>0$. If $x^{+}$ is close to $x_{s}^{s *}$ (and the decrease could potentially go to zero), then $\beta>0$ is proven by showing that the steady-state can be moved towards the target steady-state using the auxiliary control law. Let $\alpha \in(0,1)$ and $\delta>0$ be constants satisfying the conditions in Lemma IV.2, as well as the additional condition $\left\{x \mid\left\|x-x_{s}\right\|_{P} \leq \delta\right\} \subset \mathcal{E}_{f}^{s}\left(x_{s}, u_{s}\right) \forall\left(x_{s}, u_{s}\right) \in \mathcal{S}^{s}$.

Case 1: $\left\|x^{+}-x_{s}^{*}\right\|_{P} \geq \delta$

By the definition of $x^{+},\|x\|_{P}=\left\|P^{\frac{1}{2}} x\right\|_{2}$ and $x_{s}^{a}=A x_{s}^{a}+$ $B u_{s}^{a}$, we obtain: $\delta \leq\left\|A\left(x-x_{s}^{s *}\right)+B\left(u_{0}^{s *}-u_{s}^{s *}\right)\right\|_{P} \leq$ $\|A\|_{P}\left\|x-x_{s}^{s *}\right\|_{2}+\|B\|_{P}\left\|u_{0}^{s *}-u_{s}^{s *}\right\|_{2}$. Therefore either

$$
\begin{aligned}
& \|B\|_{P}\left\|u_{0}^{s *}-u_{s}^{s *}\right\|_{2} \leq 0.5 \delta \Rightarrow\|A\|_{P}\left\|x-x_{s}^{s *}\right\|_{2} \geq 0.5 \delta \\
& \Rightarrow\left\|x-x_{s}^{s *}\right\|_{Q}^{2} \geq \bar{\delta}^{2}, \\
& \text { or }\|B\|_{P}\left\|u_{0}^{s *}-u_{s}^{s *}\right\|_{2} \geq 0.5 \delta \Rightarrow\left\|u_{0}^{s *}-u_{s}^{s *}\right\|_{R}^{2} \geq \bar{\delta}^{2},
\end{aligned}
$$

where $\bar{\delta}=\frac{0.5 \delta}{\sqrt{c_{2}} \max \left(\|A\|_{P},\|B\|_{P}\right)}$ and $c_{2} \geq 1$ is such that $c_{2} Q \succeq$ $I, c_{2} R \succeq I$. From this and (12) we then get $\Delta V \leq-\beta \| x-$ $x_{r} \|_{P}^{2}$ with $\beta=\min _{x \in \mathcal{X}_{N}^{s}}\left(\left(\left\|x-x_{s}^{s *}\right\|_{Q}^{2}+\left\|u_{0}^{s *}-u_{s}^{s *}\right\|_{R}^{2}\right) / \| x-\right.$ $\left.x_{r} \|_{Q}^{2}\right)$ and therefore $\beta \geq \min _{x \in \mathcal{X}_{N}^{s}}\left(\bar{\delta}^{2} /\left\|x-x_{r}\right\|_{Q}^{2}, 1\right)>0$.

Case 2: $\left\|x^{+}-x_{s}^{*}\right\|_{P} \leq \delta$

In this case, $x^{+} \in \mathcal{E}_{f}^{s}\left(x_{s}^{*}, u_{s}^{*}\right)$, and the optimal sequence to regulate the system to the steady-state $\left(x_{s}^{*}, u_{s}^{*}\right)$ is by applying the auxiliary control law $\kappa_{f}^{t r}(x)=u_{s}^{*}+K\left(x-x_{s}^{*}\right)$.

Case 2a: $(1-\alpha)\left\|x_{s}^{s^{*}}-x_{r}\right\|_{P} \leq\left\|x^{+}-x_{s}^{*}\right\|_{P} \leq \delta$

Following similar arguments as in Case 1 it can be shown that $(1-\alpha)\left\|x_{s}^{s *}-x_{r}\right\|_{P} \leq\|A\|_{P}\left\|x-x_{s}^{s *}\right\|_{2}+\|B\|_{P}\left\|u_{0}^{s *}-u_{s}^{s *}\right\|_{2}$ and by recalling that $\|\cdot\|_{Q}^{2} \leq\|\cdot\|_{P}^{2}$ there exists a constant $\bar{\delta} \in(0,1]$ such that $\bar{\delta}^{2}(1-\alpha)^{2}\left\|x_{s}^{s *}-x_{r}\right\|_{Q}^{2} \leq\left\|x-x_{s}^{s *}\right\|_{Q}^{2}+$ $\left\|u_{0}^{s *}-u_{s}^{s *}\right\|_{R}^{2}$. From this and (12) we get

$$
\begin{aligned}
\Delta V & \leq-\frac{1}{2}\left\|x-x_{s}^{s *}\right\|_{Q}^{2}-\frac{1}{2} \bar{\delta}^{2}(1-\alpha)^{2}\left\|x_{s}^{s *}-x_{r}\right\|_{P}^{2} \\
& \leq-\frac{1}{4} \bar{\delta}^{2}(1-\alpha)^{2}\left\|x-x_{r}\right\|_{Q}^{2}
\end{aligned}
$$


and therefore $\beta=\frac{1}{4} \bar{\delta}^{2}(1-\alpha)^{2}>0$.

Case 2b: $\left\|x^{+}-x_{s}^{s *}\right\|_{P} \leq(1-\alpha)\left\|x_{s}^{s *}-x_{r}\right\|_{P} \leq \delta$

In this case we can use result (13) in Lemma IV.2. Let $\mathbf{u}^{a}, \mathbf{x}^{a}$ be the input and state sequence generated by applying the auxiliary control law $\kappa_{f}^{t r}(x)=u_{s}^{s *}+K\left(x-x_{s}^{s *}\right)$ starting from $x^{+}$and let $\epsilon^{a}$ be the associated slacks. We denote $x_{s, \alpha}=\alpha x_{s}^{s *}+(1-\alpha) x_{r}$ and similarly for $u_{s, \alpha}$. We show that $\alpha<1$, i.e. moving the steady-state $x_{s}^{s *}$ towards $x_{r}$, is feasible and provides the required cost decrease. Feasibility reduces to showing satisfaction of the terminal constraint:

$$
\begin{aligned}
& \left\|x_{N}^{a}-x_{s, \alpha}\right\|_{P}=\left\|x_{N}^{a}-x_{s}^{s *}+(1-\alpha)\left(x_{s}^{s *}-x_{r}\right)\right\|_{P} \\
& \leq\left\|(A+B K)^{N}\left(x^{+}-x_{s}^{s *}\right)\right\|_{P}+(1-\alpha)\left\|x_{s}^{s *}-x_{r}\right\|_{P} \leq \delta,
\end{aligned}
$$

which is satisfied for some $\alpha<1$, since $\|(A+B K)^{N}\left(x^{+}-\right.$ $\left.x_{s}^{s *}\right) \|_{P}<\delta$, proving that $x_{N}^{a} \in \mathcal{E}_{f}^{s}\left(x_{s, \alpha}, u_{s, \alpha}\right)$ by the definition of $\delta$.

In order to show the decrease, i.e. $\beta>0$, we first prove that the auxiliary control law provides a lower cost than the shifted sequence. By optimality of the auxiliary control law for regulation to $\left(x_{s}^{s *}, u_{s}^{s *}\right)$, we have $V_{N}\left(\mathbf{x}^{a}, \mathbf{u}^{a}, z_{s}^{*}, z_{r}\right) \leq$ $V_{N}\left(\mathbf{x}^{\text {shift }}, \mathbf{u}^{\text {shift }}, z_{s}^{*}, z_{r}\right)$. From $\left\|x_{i}^{a}-x_{s}^{s *}\right\|_{P} \leq\left\|x^{+}-x_{s}^{s *}\right\|_{\rho} \leq$ $\delta$, the condition on $\delta$ in Lemma IV.2, i.e. $2 \gamma \delta c \leq \frac{\xi}{1+\xi} f_{x}$, where $T \preceq \gamma^{2} P$, and $(1+\xi) G_{x} x_{s}^{*} \leq f_{x}+\epsilon_{s}^{s *}$, we obtain

$$
c\left\|x_{i}^{a}-x_{s}^{s *}\right\|_{T} \leq \frac{1}{2}\left(f_{x}-G_{x} x_{s}^{*}+\frac{1}{1+\xi} \epsilon_{s}^{s *}\right) \forall i=1, \ldots, N .
$$

As a result, $\epsilon_{s}^{s a}=\epsilon_{s}^{s *}, \epsilon_{i}^{s a}=0$ is feasible for $\mathbf{x}^{a}$, providing that $\boldsymbol{\epsilon}^{a} \leq \boldsymbol{\epsilon}^{\text {shift }}$ and hence $V_{N}^{s}\left(\mathbf{x}^{a}, \mathbf{u}^{a}, z_{s}^{a}, \boldsymbol{\epsilon}^{a}, z_{r}\right) \leq$ $V_{N}^{s}\left(\mathbf{x}^{\text {shift }}, \mathbf{u}^{\text {shift }}, z_{s}^{*}, \boldsymbol{\epsilon}^{\text {shift }}, z_{r}\right)$. It then follows from optimality of $V_{N}^{s}\left(x^{+}, z_{r}\right)$, Lemma IV.1 and IV.2 that

$$
\begin{aligned}
\Delta V \leq & V_{N}^{s}\left(\mathbf{x}^{a}, \mathbf{u}^{a}, z_{s, \alpha}^{*}, \boldsymbol{\epsilon}_{\alpha}^{a}, z_{r}\right)-V_{N}^{s *}\left(x, z_{r}\right) \\
\leq & V_{N}^{s}\left(\mathbf{x}^{a}, \mathbf{u}^{a}, z_{s}^{*}, \boldsymbol{\epsilon}^{a}, z_{r}\right)-V_{N}^{s *}\left(x, z_{r}\right) \\
& -(1-\alpha)^{2}\left\|x_{s}^{s *}-x_{r}\right\|_{P}^{2} \\
\leq & V_{N}^{s}\left(\mathbf{x}^{\text {shift }}, \mathbf{u}^{\text {shift }}, z_{s}^{*}, \boldsymbol{\epsilon}^{\text {shift }}, z_{r}\right)-V_{N}^{s *}\left(x, z_{r}\right) \\
& -(1-\alpha)^{2}\left\|x_{s}^{s *}-x_{r}\right\|_{P}^{2} \\
\leq & -\left\|x-x_{s}^{*}\right\|_{Q}^{2}-(1-\alpha)^{2}\left\|x_{s}^{s *}-x_{r}\right\|_{P}^{2} \\
\leq & -\frac{1}{2}(1-\alpha)^{2}\left\|x-x_{s}^{s *}\right\|_{Q}^{2}
\end{aligned}
$$

i.e. $\beta=\frac{1}{2}(1-\alpha)^{2}>0$.

A decrease of the Lyapunov function with $\beta>0$ is therefore guaranteed in all cases, which concludes the proof.

\section{REFERENCES}

[1] D. Q. Mayne, J. B. Rawlings, C. V. Rao, and P. O. M. Scokaert, "Constrained model predictive control: Stability and optimality," Automatica, vol. 36(6), pp. 789-814, 2000.

[2] E. Zafiriou and H.-W. Chiou, "Output constraint softening for SISO model predictive control," in Proc. of the American Control Conference, 1993, pp. 372-376.

[3] A. Zheng and M. Morari, "Stability of model predictive control with mixed constraints," IEEE Transactions on Automatic Control, vol. 40, no. 10, pp. 1818-1823, 1995.

[4] P. O. M. Scokaert and J. B. Rawlings, "Feasibility issues in linear model predictive control," AIChE Journal, vol. 45, pp. 1649-1659, 1999.

[5] N. M. C. de Oliveira and L. T. Biegler, "Constraint handling and stability properties of model-predictive control," AIChE Journal, vol. 40, pp. 1138-1155, July 1994.
[6] E. C. Kerrigan and J. M. Maciejowski, "Soft constraints and exact penalty functions in model predictive control," in Proc. UKACC International Conference (Control), Cambridge, UK, Sept. 2000.

[7] S. C. Thomsen, H. Niemann, and N. K. Poulsen, "Robust stability in predictive control with soft constraints," in Proc. of the American Control Conference, 2010, pp. 6280-6285.

[8] A. G. Wills and W. P. Heath, "Barrier function based model predictive control," Automatica, vol. 40, pp. 1415-1422, 2004.

[9] D. Limon, I. Alvarado, T. Alamo, and E. F. Camacho, "MPC for tracking piecewise constant references for constrained linear systems," Automatica, vol. 44, pp. 2382-2387, 2008.

[10] S. Boyd and L. Vandenberghe, Convex Optimization. Cambridge University Press, 2004.

[11] M. Andersen, J. Dahl, Z. Liu, and L. Vandenberghe, "Interior-point methods for large-scale cone programming," in Optimization for Machine Learning. MIT Press, 2011, pp. 55-83.

[12] E. D. Andersen, C. Roos, and T. Terlaky, "On implementing a primaldual interior-point method for conic quadratic optimization," Mathematical Programming, vol. 95, pp. 249-277, 2003.

[13] A. Domahidi, E. Chu, and S. Boyd, "ECOS: An SOCP solver for embedded systems," in Proc. of the 2013 European Control Conference, July 2013, pp. 3071-3076.

[14] M. N. Zeilinger, C. N. Jones, and M. Morari, "Robust stability properties of soft constrained MPC," in Proc. of the 49th IEEE Conf. on Decision and Control, 2010, pp. 5276-5282.

[15] M. Vidyasagar, Nonlinear Systems Analysis, 2nd ed. Prentice Hall, 1993.

[16] F. Blanchini, "Set invariance in control," Automatica, vol. 35, pp. 1747$1767,1999$.

[17] Z.-P. Jiang and Y. Wang, "Input-to-state stability for discrete-time nonlinear systems," Automatica, vol. 37, pp. 857-869, 2001.

[18] E. D. Sontag and Y. Wang, "New characterizations of input-to-state stability," IEEE Transactions on Automatic Control, vol. 41, pp. 12831294, 1999.

[19] D. Limon, T. Alamo, D. M. Raimondo, D. Muñoz de la Peña, J. M. Bravo, A. Ferramosca, and E. F. Camacho, "Input-to-state stability: A unifying framework for robust model predictive control," Lecture Notes in Control and Information Sciences, vol. 384, pp. 1-26, 2009.

[20] J. B. Rawlings and D. Q. Mayne, Model Predictive Control: Theory and Design. Nob Hill Publishing, 2009.

[21] J. Maciejowski, Predictive Control with Constraints. Prentice Hall, 2000.

[22] A. Ferramosca, D. Limon, I. Alvarado, T. Alamo, and E. F. Camacho, "MPC for tracking with optimal closed-loop performance," Automatica, vol. 45, pp. 1975-1978, August 2009.

[23] D. Limon, I. Alvarado, T. Alamo, and E. F. Camacho, "Robust tubebased MPC for tracking of constrained linear systems with additive disturbances," Journal of Process Control, vol. 20, no. 3, pp. 248-260, March 2010.

[24] S. Boyd, L. El Ghaoui, E. Feron, and V. Balakrishnan, Linear Matrix Inequalities in System and Control Theory, ser. Studies in Applied Mathematics. Philadelphia, PA: SIAM, Jun. 1994, vol. 15.

[25] R. Fletcher, Practical Methods of Optimization, 2nd ed. John Wiley and Sons, New York, 1987.

[26] M. N. Zeilinger, D. M. Raimondo, A. Domahidi, M. Morari, and C. N. Jones, "On Real-time Robust Model Predictive Control," 2014, Automatica. To appear.

[27] G. Grimm, M. J. Messina, S. E. Tuna, and A. R. Teel, "Examples when nonlinear model predictive control is nonrobust," Automatica, vol. 40, no. 10, pp. 1729-1738, 2004

[28] D. Q. Mayne, M. M. Seron, and S. V. Rakovic, "Robust model predictive control of constrained linear systems with bounded disturbances," Automatica, vol. 41, no. 2, pp. 219-234, 2005.

[29] B. Bank, J. Guddat, D. Klatte, B. Kummer, and K. Tammer, Non-linear parametric optimization. Akademie-Verlag, Berlin, 1982.

[30] M. N. Zeilinger, "Real-time Model Predictive Control," Ph.D. dissertation, ETH Zurich, 2011.

[31] J. Löfberg, "YALMIP : A Toolbox for Modeling and Optimization in MATLAB," in Proc. of the 2004 IEEE Int. Symp. on Computer Aided Control Systems Design, 2004, pp. 284-289.

[32] M. Kvasnica, Real-Time Model Predictive Control via Multi-Parametric Programming: Theory and Tools. VDM Verlag, 2009. 


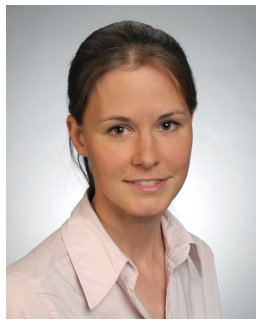

Melanie N. Zeilinger Melanie N. Zeilinger received the diploma in Engineering Cybernetics from the University of Stuttgart, Germany, in 2006. She conducted her diploma thesis research at the Department of Chemical Engineering, University of California at Santa Barbara, USA, in 2005-2006. In 2011 she received the Dr.sc. degree with honors in Electrical Engineering from ETH Zurich, Switzerland. From 2011-2012 she was a postdoctoral fellow in the Automatic Control Laboratory at the École Polytechnique Fédérale de Lausanne (EPFL), Switzerland. She is currently a Marie Curie fellow at the Max-Planck Institute for Intelligent Systems, Tübingen, Germany, and a postdoctoral researcher at the University of California, Berkeley, USA. Her current research interests include real-time and distributed control and optimization and the development of modeling and control techniques for green energy-efficient technologies.

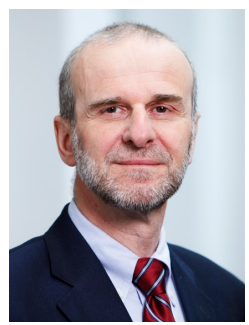

Manfred Morari Manfred Morari was head of the Department of Information Technology and Electrical Engineering at ETH Zurich from 2009 to Jan2012. He was head of the Automatic Control Laboratory from 1994 to 2008 . Before that he was the McCollum-Corcoran Professor of Chemical Engineering and Executive Officer for Control and Dynamical Systems at the California Institute of Technology. He obtained the diploma from ETH Zurich and the Ph.D. from the University of Minnesota, both in chemical engineering. His interests are in hybrid systems and the control of biomedical systems. In recognition of his research contributions he received numerous awards, among them the Donald P. Eckman Award, the John R. Ragazzini Award and the Richard E. Bellman Control Heritage Award of the American Automatic Control Council, the Allan P. Colburn Award and the Professional Progress Award of the AIChE, the Curtis W. McGraw Research Award of the ASEE, Doctor Honoris Causa from Babes-Bolyai University, Fellow of IEEE, IFAC and AIChE, the IEEE Control Systems Technical Field Award, and was elected to the National Academy of Engineering (U.S.). Manfred Morari has held appointments with Exxon and ICI plc and serves on the technical advisory boards of several major corporations.

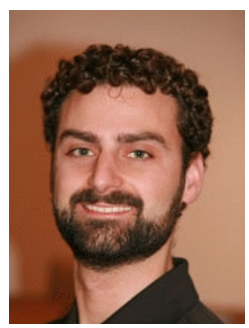

Colin N. Jones Colin N. Jones is an Assistant Professor in the Automatic Control Laboratory at the École Polytechnique Fédérale de Lausanne (EPFL) in Switzerland. He was a Senior Researcher at the Automatic Control Lab at ETH Zurich until 2010 and obtained a Ph.D. in 2005 from the University of Cambridge for his work on polyhedral computational methods for constrained control. Prior to that, he was at the University of British Columbia in Canada, where he took a bachelor and master in Electrical Engineering and Mathematics. His current research interests are in the areas of high-speed predictive control and optimization, as well as green energy generation, distribution and management. 